BULLETIN (New Series) OF THE AMERICAN MATHEMATICAL SOCIETY

Volume 36, Number 1, January 1999, Pages 1-26

S 0273-0979(99)00766-1

\title{
ZEROES OF ZETA FUNCTIONS AND SYMMETRY
}

\author{
NICHOLAS M. KATZ AND PETER SARNAK
}

\begin{abstract}
Hilbert and Polya suggested that there might be a natural spectral interpretation of the zeroes of the Riemann Zeta function. While at the time there was little evidence for this, today the evidence is quite convincing. Firstly, there are the "function field" analogues, that is zeta functions of curves over finite fields and their generalizations. For these a spectral interpretation for their zeroes exists in terms of eigenvalues of Frobenius on cohomology. Secondly, the developments, both theoretical and numerical, on the local spacing distributions between the high zeroes of the zeta function and its generalizations give striking evidence for such a spectral connection. Moreover, the low-lying zeroes of various families of zeta functions follow laws for the eigenvalue distributions of members of the classical groups. In this paper we review these developments. In order to present the material fluently, we do not proceed in chronological order of discovery. Also, in concentrating entirely on the subject matter of the title, we are ignoring the standard body of important work that has been done on the zeta function and $L$-functions.
\end{abstract}

\section{The Montgomery-Odlyzko LaW}

We begin with the Riemann Zeta function and some phenomenology associated with it.

$$
\zeta(s)=\sum_{n=1}^{\infty} n^{-s}=\prod_{p}\left(1-p^{-s}\right)^{-1}
$$

the product being over the primes, and it converges for $\operatorname{Re}(s)>1$. As was shown by Riemann $[\mathrm{RI}] \zeta(s)$ has a continuation to the complex plane and satisfies a functional equation

$$
\xi(s):=\pi^{-s / 2} \Gamma(s / 2) \zeta(s)=\xi(1-s) ;
$$

$\xi(s)$ is entire except for simple poles at $s=0$ and 1 . We write the zeroes of $\xi(s)$ as

$$
\frac{1}{2}+i \gamma
$$

From (1) it is clear that $|\operatorname{Im}(\gamma)| \leq 1 / 2$. Hadamard and de la Vallee Poisson in their (independent) proofs of the Prime Number Theorem established that $|\operatorname{Im}(\gamma)|<1 / 2$. The well known Riemann Hypothesis "RH" asserts that $\gamma \in \mathbb{R}$. In what follows we are interested in finer questions about the distribution of the zeroes. Let's assume

Received by the editors October 15, 1997, and in revised form August 28, 1998.

1991 Mathematics Subject Classification. Primary 11G, 11M, 11R, 11Y; Secondary 60B, 81Q. Research partially supported by NSF grants DMS 9506412 and DMS 9401571.

(C)1999 American Mathematical Society 


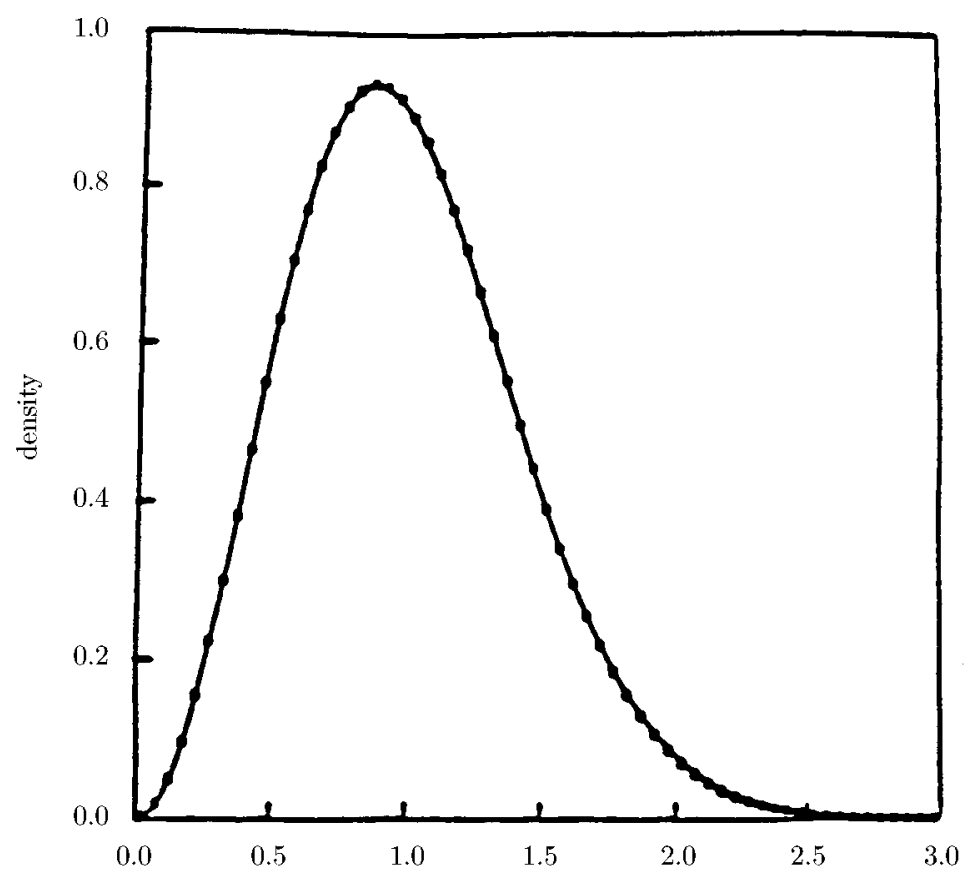

FiguRE 1. Nearest neighbor spacings among 70 million zeroes beyond the $10^{20}$-th zero of zeta, verses $\mu_{1}$ (GUE).

$\mathrm{RH}$ (needless to say, in the numerical experiments reported on below all zeroes found were on the line $\left.\operatorname{Re}(s)=\frac{1}{2}\right)$ and order the ordinates $\gamma$ :

$$
\ldots \ldots \gamma_{-1} \leq 0 \leq \gamma_{1} \leq \gamma_{2} \ldots
$$

Then $\gamma_{j}=-\gamma_{-j}, j=1,2, \ldots$, and in fact $\gamma_{1}$ is rather large, being equal to $14.1347 \ldots$. It is known (apparently already to Riemann) that

$$
\#\left\{j: 0 \leq \gamma_{j} \leq T\right\} \sim \frac{T \log T}{2 \pi}, \text { as } T \rightarrow \infty .
$$

In particular, the mean spacing between the $\gamma_{j}^{\prime} s$ tends to zero as $j \rightarrow \infty$. In order to examine the (statistical) law of the local spacings between these numbers we re-normalize (or "unfold" as it is sometimes called) as follows: Set

$$
\widehat{\gamma}_{j}=\frac{\gamma_{j} \log \gamma_{j}}{2 \pi} \quad \text { for } \quad j \geq 1
$$

The consecutive spacings $\delta_{j}$ are defined to be

$$
\delta_{j}=\widehat{\gamma}_{j+1}-\widehat{\gamma}_{j}, j=1,2, \ldots
$$

More generally, the $k-t h$ consecutive spacings are

$$
\delta_{j}^{(k)}=\widehat{\gamma}_{j+k}-\widehat{\gamma}_{j}, j=1,2, \ldots
$$

What laws (i.e. distributions), if any, do these numbers obey?

During the years 1980-present, Odlyzko [OD] has made an extensive and profound numerical study of the zeroes and in particular their local spacings. He finds that they obey the laws for the (scaled) spacings between the eigenvalues of 
a typical large unitary matrix. That is they obey the laws of the Gaussian (or equivalently, Circular) Unitary Ensemble GUE (see Section 2 for definitions). In Figure 1, the histogram of the spacings $\delta_{j}$ for $10^{20} \leq j \leq 10^{20}+7.10^{6}$ is plotted against the GUE prediction ( $\mu_{1}$ (GUE) - the Gaudin distribution of Section 2). At the phenomenological level this is perhaps the most striking discovery about zeta since Riemann. The big questions, which we attempt to answer here, are, why is this so and what does it tell us about the nature (e.g. spectral) of the zeroes? Also, what is the symmetry behind this "GUE" law?

Odlyzko's computations were inspired by the 1974 discovery of Montgomery [MO2] that the "pair correlation" of the zeroes is, at least for a restricted class of test functions, equal to the GUE pair correlation $R_{2}$ (GUE) (see Section 2). Precisely, he proves that for any $\phi \in \mathcal{S}(\mathbb{R})$ for which the support of $\hat{\phi}(\xi)=\int_{-\infty}^{\infty} \phi(x) e^{-2 \pi i x \xi} d x$ is contained in $(-1,1)$

$$
\lim _{N \rightarrow \infty} \frac{1}{N} \sum_{1 \leq j \neq k \leq N} \phi\left(\widehat{\gamma}_{j}-\widehat{\gamma}_{k}\right)=\int_{-\infty}^{\infty} \phi(x) r_{2}(\mathrm{GUE})(x) d x
$$

where

$$
r_{2}(\operatorname{GUE})(x)=1-\left(\frac{\sin \pi x}{\pi x}\right)^{2}
$$

The significance of the interval $(-1,1)$ is that $r_{2} \widehat{(\mathrm{GUE})}(\xi)$ changes its analytic character at $\xi= \pm 1$, and this signals that for $\hat{\phi}^{\prime} s$ whose support is outside $[-1,1]$ there will be new "non-diagonal" (the main contribution to the limit in (9) for restricted $\phi$ comes from the diagonal) terms contributing to the main term. Montgomery conjectured that (9) holds without any restrictions on the support of $\hat{\phi}$, and in [GO-MO] he and Goldston give an equivalence of this conjecture in terms of prime numbers. In Figure 2 a comparison of (9) for $\phi^{\prime} s$ which are characteristic functions of small intervals (i.e. the histogram) with $R_{2}(\mathrm{GUE})$ is displayed.

One can look at triple and higher generalizations of the left hand side of (9); see [R-S]. Indeed, the knowledge of the $n$-level correlations for all $n$ determines all the local spacing laws (see [K-S1]) and in particular the $k$-th consecutive spacings. In [HE], Hejhal, using Montgomery's method, established that the triple correlation is the GUE triple correlation as determined by Dyson [DY]. Rudnick and Sarnak [R-S] by a somewhat different method (which in fact does not appeal to $\mathrm{RH}$ ) establish that all the $n \geq 2$ correlations are as predicted by GUE. All of these results are restricted as in (9); that is they are proven only for test functions whose Fourier Transforms are restricted so that only the "diagonal" terms are responsible for the main term. A heuristic derivation of the $n$-level correlations without any restrictions on the Fourier Transform has been given by Bogomolny and Keating [B-K]. The calculations of the correlations above are based on the "explicit formula" (see [R-S]) which allows one to express the correlations in terms of multiple sums over primes. The combinatorics which take one from these sums over primes to the GUE $n$-level correlations are fascinating but hardly illuminating in connection with gaining any deeper insight relating the zeroes and GUE.

The Riemann Zeta Function is but the first of a zoo of zeta and $L$-functions for which we can ask similar questions. There are the Dirichlet $L$-functions $L(s, \chi)$ defined as follows: $q \geq 1$ is an integer, $\chi:(\mathbb{Z} / q \mathbb{Z})^{*} \rightarrow \mathbb{C}^{*}$ a (primitive) character 


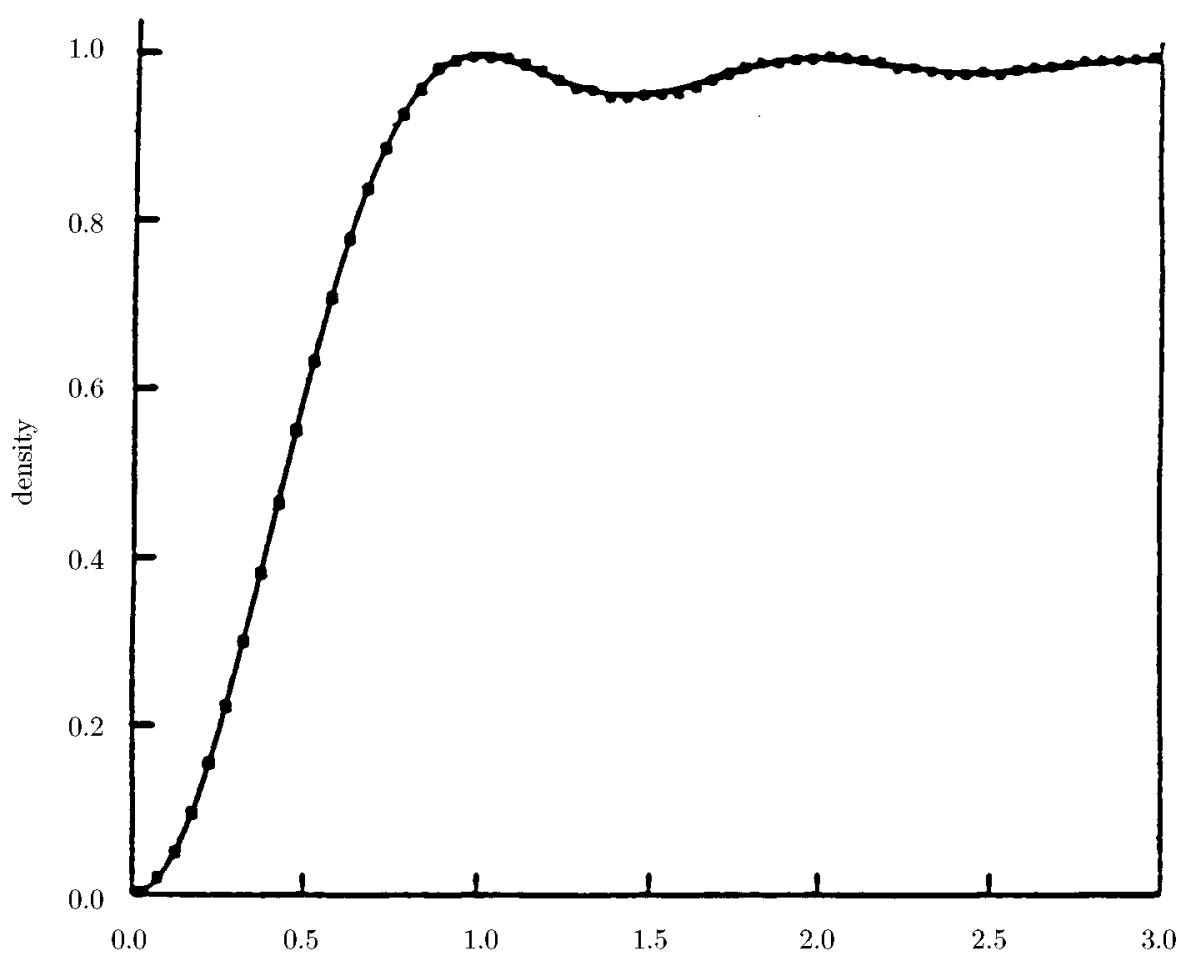

Figure 2. Pair correlation for zeros of zeta based on $8 \times 10^{6}$ zeros near the $10^{20}$-th zero, versus the GUE conjectured density 1 $\left(\frac{\sin \pi x}{\pi x}\right)^{2}$.

and we extend $\chi$ to $\mathbb{Z}$ by making it periodic, and $\chi(m)=0$ if $(m, q) \neq 1$. Then

$$
L(s, \chi)=\sum_{n=1}^{\infty} \chi(n) n^{-s}=\prod_{p}\left(1-\chi(p) p^{-s}\right)^{-1} .
$$

The analogue of (2) is known:

$$
\xi(s, \chi):=\pi^{-s / 2} \Gamma\left(\frac{s+a_{\chi}}{2}\right) L(s, \chi)=q^{s-\frac{1}{2}} \epsilon_{\chi} \xi(1-s, \bar{\chi})
$$

where $a_{\chi}=0$ if $\chi(-1)=1$ and is 1 if $\chi(-1)=-1$, while $\left|\epsilon_{\chi}\right|=1$ and $\epsilon_{\chi}$ is in fact a unitarized Gauss sum. $q$ is called the conductor of $\chi$. The proof of (11') is the same as for zeta and is based on Poisson summation [DA]. More generally by the work of Godement and Jacquet [JA] if $f$ is an automorphic cusp form on $G L_{m} / \mathbb{Q}, m \geq 1$, its (standard) $L$-function $L(s, f)$ is entire (if $f$ is not the trivial representation on $G L_{1}$ ) and satisfies a functional equation similar to (2) (with an appropriate conductor and $\epsilon$-factor). Such an $L$-function, $L(s, f)$, is given by an Euler product of degree $m$ :

$$
L(s, f)=\prod_{p} L\left(s, f_{p}\right)
$$


where

$$
L\left(s, f_{p}\right)=\prod_{j=1}^{m}\left(1-\alpha_{j, f}(p) p^{-s}\right)^{-1}
$$

The $\alpha_{j, f}(p)^{\prime} s$ are eigenvalues of local (at $p$ ) Hecke algebra's acting on $f$. In all these cases $L(s, f)$ is expected to satisfy $\mathrm{RH}$; that is its non-trivial zeroes are on the line $\operatorname{Re}(s)=1 / 2$. General Conjectures of Langlands [LA] assert that all $L$-functions are finite products of these standard (cuspidal) $L$-functions, $L(s, f)$.

A classical and concrete example of a form on $G L_{2} / \mathbb{Q}$ is $f=\Delta$ :

$$
\Delta(q):=q \prod_{n=1}^{\infty}\left(1-q^{n}\right)^{24}=\sum_{n=1}^{\infty} \tau(n) q^{n} .
$$

With $q=e^{2 \pi i z}, \Delta(z)$ is a holomorphic cusp form of weight 12 for $\Gamma=S L_{2}(\mathbb{Z})$. That is for $z \in \mathbb{H}$, the upper half plane, we have

$$
\Delta\left(\frac{a z+b}{c z+d}\right)=(c z+d)^{12} \Delta(z)
$$

for all $a, b, c, d \in \mathbb{Z}, a d-b c=1$.

Its $L$-function is

$$
L(s, \Delta)=\sum_{n=1}^{\infty} \frac{\tau(n)}{n^{11 / 2}} n^{-s}=\prod_{p}\left(1-\frac{\tau(p)}{p^{11 / 2}} p^{-s}+p^{-2 s}\right)^{-1},
$$

and it is entire and satisfies

$$
\xi(s, \Delta)=(2 \pi)^{-s} \Gamma\left(s+\frac{11}{2}\right) L(s, \Delta)=\xi(1-s, \Delta) .
$$

Other basic examples in $G L_{2} / \mathbb{Q}$ are $L(s, E)$ where $E / \mathbb{Q}$ is an elliptic curve [SI1]. The prescription of the local (degree 2) factor at each prime $p$ is given in terms of an analysis of $E$ over $\mathbb{F}_{p}$ (see [SI1]). A well known conjecture of Shimura and Taniyama, first formulated precisely in Weil [WE1], asserts that $L(s, E)=L(s, f)$

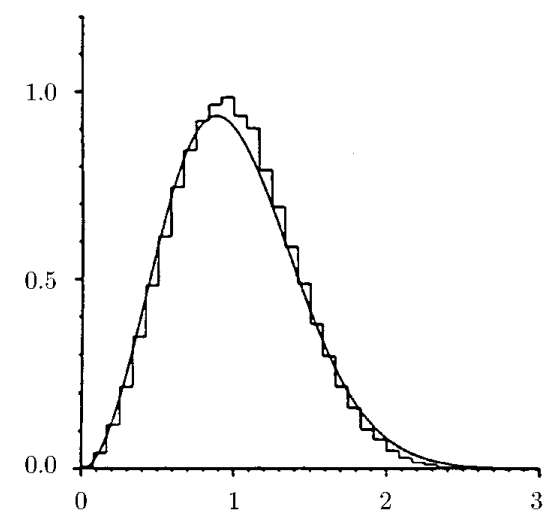

FiguRE 3. Nearest neighbor spacings distribution for the Ramanujan $L$-function, $N=$ 138693.

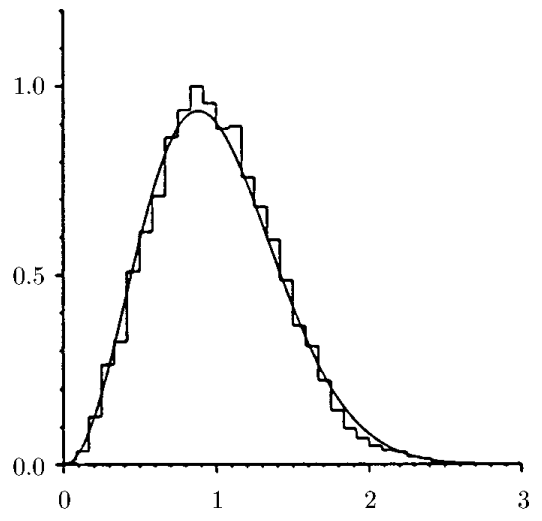

Figure 4. Nearest neighbor spacings distribution for the $L$ function associated to $E: y^{2}+$ $y=x^{3}-x, N=5374$. 
where $f$ is a holomorphic cusp form of weight 2 for $\Gamma_{0}(N)$ where $N$ is the conductor of $E$ (see [SI1]). Here $\Gamma_{0}(N)=\left\{\left[\begin{array}{ll}a & b \\ c & d\end{array}\right] \in S L_{2}(\mathbb{Z}): N \mid c\right\}$ and $f(z)$ satisfies:

$$
f(\gamma z)=(c z+d)^{2} f(z), \gamma \in \Gamma_{0}(N) .
$$

The results of Rudnick and Sarnak [R-S] were carried out in the general context of $f$ being an automorphic cusp form for $G L_{m} / \mathbb{Q}$. They show that the $n \geq 2$ correlations of the zeroes of $L(s, f)$ (again in restricted ranges) are universally the GUE ones! Numerical experimentation by Rumely [RUM] for Dirichlet $L$-functions and by Rubinstein $[\mathrm{RUB}]$ for a variety of $G L_{2} / \mathbb{Q}$ forms $f$ strongly confirm this universality (so in particular confirm that no restrictions on the test functions are needed). For example, the consecutive spacings for the zeroes of $L(s, \Delta)$ versus $\mu_{1}(\mathrm{GUE})$ are given in Figure 3 and similarly for an $L(s, E)$ in Figure 4.

We call this phenomenon - that the high zeroes of any fixed $L(s, f), f$ a cusp form on $G L_{m} / \mathbb{Q}$ obey GUE spacing laws - the "Montgomery-Odlyzko Law".

\section{RANDOM MATRIX MODELS}

In the 50's (see [WI] ) Wigner suggested that the resonance lines of a heavy nucleus (their determination by analytic means being intractable) might be modeled by the spectrum of a large random matrix. To this end he considered various ensembles (i.e. probability distributions) on spaces of matrices: in particular, the Gaussian Orthogonal Ensemble, 'GOE', and Gaussian Unitary Ensemble, 'GUE'. These live on the linear space of real symmetric (resp. hermitian) $N \times N$ matrices and are orthogonal (resp. unitary) invariant ensembles. He raised the question of the local (scaled) spacing distributions between the eigenvalues of typical members of these ensembles as $N \rightarrow \infty$. The answer was provided by Gaudin [GA] and Gaudin-Mehta [G-M], who make ingenious use of orthogonal polynomials. This technique is a key tool in the derivation of the results below. Later Dyson [DY] introduced his three closely related circular ensembles: COE, CUE, as well as CSE with it's associated Gaussian Symplectic Ensemble, 'GSE'. These circular ensembles may be realized as the compact Riemannian symmetric spaces (with their volume form as probability measure) $U(N) / O(N), U(N)$ and $U(2 N) / U S p(2 N)$, respectively. He investigated the local spacing statistics for the eigenvalues of the matrices in these ensembles (in their standard realization, see Table 1 below) as $N \rightarrow \infty$. He shows that these statistics agree with the corresponding matrices from the GOE, GUE and GSE ensembles.

Now the above are but 3 of the 11 classical compact irreducible symmetric spaces (we ignore the center $U(1)$ of $U(N)$ which in the limit $N \rightarrow \infty$ plays no role) of Cartan (see [HEL] ). That some of these other matrix models are of importance in the theory of $L$-functions will become clear below. Apparently there are also some physical problems which require some of the other symmetric spaces [A-Z]. We list 6 of the 11 symmetry types: For our purposes of symmetry associated with $L$-functions, only the first 4 ensembles in Table 1 will play a role. These 4 are the classical compact groups which with a bi-invariant metric yield the so-called type II symmetric spaces (see [HEL] ). The invariant volume form on $G(N)$ is just Haar measure.

The ensemble $U(N)$ is Dyson's CUE. The non-compact dual symmetric space of $U(N)$ is $G L_{N}(\mathbb{C}) / U(N)$, which is the space on which GUE lives. Similarly, the non-compact dual of $U(N) / O(N)$ is $G L_{N}(\mathbb{R}) / O(N)$, that is GOE, and of 
TABLE 1

\begin{tabular}{|c|c|}
\hline SYMMETRY TYPE $G$ & REALIZATION OF $G(N)$ AS MATriCES \\
\hline$U$ & $U(N)$, the compact group of $N \times N$ unitary matrices. \\
\hline$S O$ (even) & $\begin{array}{l}S O(2 N) \text {, the compact group of } 2 N \times 2 N \text { unitary(!) matrices preserving } \\
\text { the orthogonal form } I \text {, i.e. unitary matrices } A \text { satisfying } A^{t} A=I \text {. }\end{array}$ \\
\hline$S O$ (odd) & $S O(2 N+1)$ and as above. \\
\hline$S p$ & $\begin{array}{l}U S p(2 N) \text {, the compact group of } 2 N \times 2 N \text { unitary matrices } A \text { satisfying } \\
A^{t} J A=J, J=\left[\begin{array}{cc}0 & I_{N} \\
-I_{N} & 0\end{array}\right] .\end{array}$ \\
\hline $\mathrm{COE}$ & $\begin{array}{l}U(N) / O(N) \text {, symmetric unitary } N \times N \text { matrices identified with the } \\
\text { above cosets via } B \rightarrow B^{t} B \text {. }\end{array}$ \\
\hline CSE & $\begin{array}{l}U(2 N) / U S p(2 N), 2 N \times 2 N \text { unitary matrices satisfying } J^{t} H^{t} J=H \\
\text { identified by } B \rightarrow B J B^{t} J^{t} \text {. }\end{array}$ \\
\hline
\end{tabular}

$U(2 N) / U S p(2 N)$ is $U^{*}(2 N) / U S p(2 N)$ (see [HEL], whose notation we adopt), which is the space for GSE.

Let $G(N)$ be any one of the ensembles in Table 1 realized as unitary matrices $A \in G(N)$. Let $d A$ denote the invariant measure and $e^{i \theta_{1}(A)}, e^{i \theta_{2}(A)}, \ldots, e^{i \theta_{N}(A)}$ the eigenvalues of $A$. We order these

$$
0 \leq \theta_{1}(A) \leq \theta_{2}(A) \ldots \leq \theta_{N}(A)<2 \pi .
$$

The local (scaled) spacing distributions between the eigenvalues of $A$ are defined as follows:

- the $k$-th consecutive spacings $\mu_{k}(A)$ are a measure on $[0, \infty)$

$$
\mu_{k}(A)[a, b]=\frac{\#\left\{1 \leq j \leq N \mid \frac{N}{2 \pi}\left(\theta_{j+k}-\theta_{j}\right) \in[a, b]\right\}}{N} .
$$

The scaling factor $N / 2 \pi$ normalizes $\mu_{k}(A)$ to have mean equal to $k$.

- The pair-correlation $R_{2}(A)$ measures the distribution between all pairs of eigenvalues of $A$. For $[a, b] \subset \mathbb{R}$ a compact interval

$$
R_{2}(A)[a, b]=\frac{\#\left\{j \neq k \mid \frac{N}{2 \pi}\left(\theta_{j}-\theta_{k}\right) \in[a, b]\right\}}{N} .
$$

Higher correlations may be defined similarly.

The main question to be answered here is the behavior of these measures as $N \rightarrow \infty$. For $G(N)$ any one of the type II symmetric spaces above, Katz and Sarnak [K-S1] establish the following:

- Fix $k \geq 1$. There are measures $\mu_{k}(\mathrm{GUE})$ such that for any $G(N)$ of type II

$$
\lim _{N \rightarrow \infty} \int_{G(N)} \mu_{k}(A) d A=\mu_{k}(\mathrm{GUE}) .
$$

- A Law of Large Numbers which ensures that for a typical (in measure) $A \in G(N), \mu_{k}(A)$ and $R_{2}(A)$ approach $\mu_{k}(\mathrm{GUE})$ and $R_{2}(\mathrm{GUE})$ as $N \rightarrow \infty$. 
Precisely

$$
\lim _{N \rightarrow \infty} \int_{G(N)} D\left(\mu_{k}(A), \mu_{k}(\mathrm{GUE})\right) d A=0
$$

where $D\left(\nu_{1}, \nu_{2}\right)$ is the Kolomogorof-Smirnov distance between $\nu_{1}$ and $\nu_{2}$; that is

$$
D\left(\nu_{1}, \nu_{2}\right)=\sup \left\{\left|\nu_{1}(I)-\nu_{2}(I)\right|: I \subset \mathbb{R} \text { an interval }\right\} .
$$

For $[a, b] \subset \mathbb{R}$ (compact)

$$
\lim _{N \rightarrow \infty} \int_{G(N)}\left|R_{2}(A)[a, b]-R_{2}(\mathrm{GUE})[a, b]\right| d A=0
$$

where

$$
R_{2}(\mathrm{GUE})[a, b]=\int_{a}^{b} r_{2}(\mathrm{GUE})(x) d x
$$

and $r_{2}(\mathrm{GUE})$ is given in (10).

Given that the answer is universal for type II symmetric spaces, and since CUE is of this type and as pointed out above CUE and GUE have the same local spacing statistics, it follows that type II local spacings are GUE (as indicated by the notation in (21) and (22)). Gaudin in the original paper [GA] expressed the measures $\mu_{k}(\mathrm{GUE})$ in terms of a Fredholm determinant:

$$
d \mu_{k}(\mathrm{GUE})=\frac{d^{2}}{d s^{2}}\left(\left.\sum_{j=0}^{k} \frac{k-j}{j !}\left(\frac{\partial}{\partial T}\right)^{j} \operatorname{det}(I+T K(s))\right|_{T=1}\right) d s
$$

where $K(s)$ is the trace class operator on $L^{2}[-s / 2, s / 2]$ whose kernel is

$$
K(x, y)=\frac{\sin \pi(x-y)}{\pi(x-y)} .
$$

He also noted that (23) allows one to compute $\mu_{k}$ numerically. Indeed, the eigenfunctions of the integral equation

$$
\int_{-s / 2}^{s / 2} K(x, y) f(y) d y=\lambda f(x)
$$

are prolate-spheroidal functions $[\mathrm{MEH}]$. One may use this to compute the eigenvalues $\lambda_{j}(s)$ and eigenfunctions $f_{j}(x, s)$ of (26) and from it the densities of the measures $\mu_{k}(\mathrm{GUE})$. The density of $\mu_{1}(\mathrm{GUE})$ is the solid curve in Figure 1. Notice that the density vanishes to second order at $s=0$, which says that the eigenvalues of a typical $A$ in a large $G(N)$ tend to "repel" each other. For the ensembles COE and CSE the analogous measures $\mu_{k}(\mathrm{COE})$ and $\mu_{k}(\mathrm{CSE})$ have been determined (see Mehta $[\mathrm{MEH}])$; they are quite different from $\mu_{k}(\mathrm{GUE})$ as well as from each other.

While the above results show that the local spacings between all the eigenvalues of a typical $A$ in any $G(N)$ of type II are universally GUE as $N \rightarrow \infty$, the distribution of the eigenvalue nearest to 1 is sensitive to the particular symmetry $G$. For $k \geq 1$, let $\nu_{k}(G(N))$ be the measure on $[0, \infty)$ which gives the distribution of the $k$-th eigenvalue of $A$, as $A$ varies over $G(N)$. That is

$$
\nu_{k}(G(N))[a, b]=\operatorname{Haar}\left\{A \in G(N) \mid \frac{\theta_{k}(A) N}{2 \pi} \in[a, b]\right\} .
$$



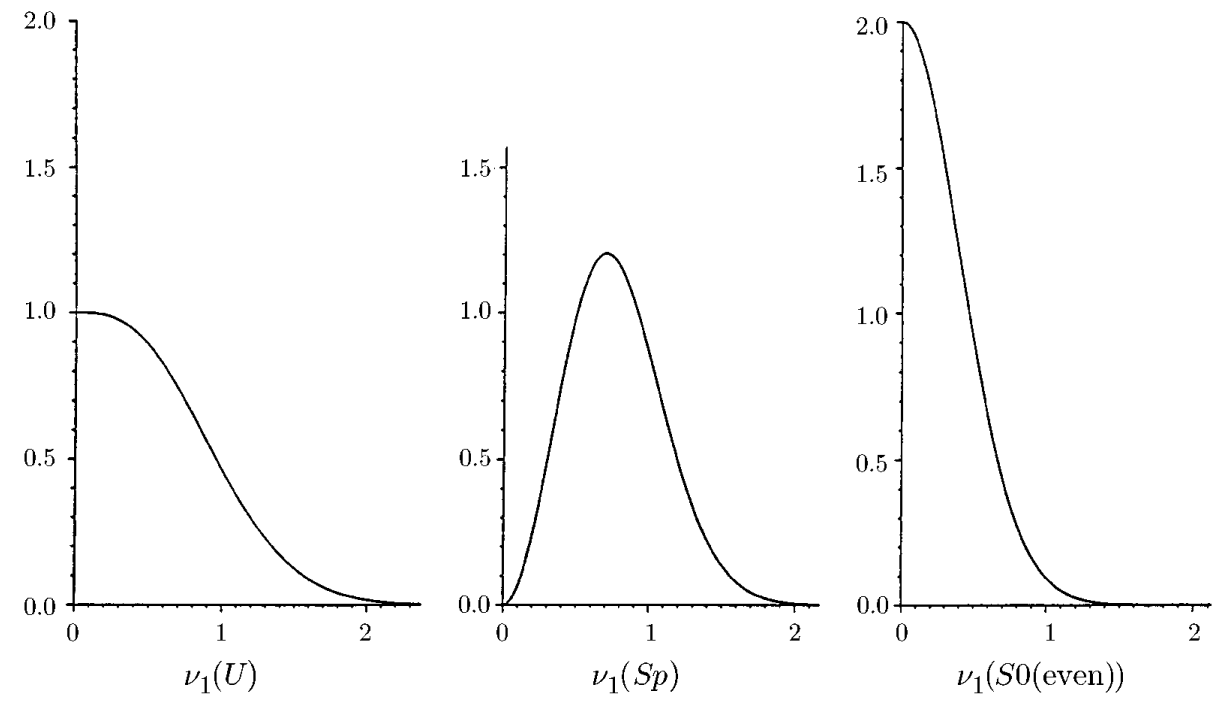

FiguRE 5

Similarly one forms the 1-level scaling density (or more generally $n$-level densities) of eigenvalues of $A$ near 1 . For such an $A \in G(N)$ and $[a, b] \subset \mathbb{R}$, let

$\Delta(A)[a, b]=\#\left\{\theta(A) \mid e^{i \theta(A)}\right.$ is an eigenvalue of $A$ and $\left.(\theta(A) N) / 2 \pi \in[a, b]\right\}$.

The average of $\Delta(A)$ is denoted by $W$; that is

$$
W(G(N))=\int_{G(N)} \Delta(A) d A .
$$

In [K-S1] it is shown that there are measures $\nu_{k}(G)$ on $[0, \infty)$ which depend on the symmetry $G$ such that

$$
\lim _{N \rightarrow \infty} \nu_{k}(G(N))=\nu_{k}(G) .
$$

For the densities we have

$$
\lim _{N \rightarrow \infty} W(G(N))[a, b]=\int_{a}^{b} w(G)(x) d x
$$

where

$$
w(G)(x)= \begin{cases}1 & \text { if } G=U \text { or } S U \\ 1-\frac{\sin 2 \pi x}{2 \pi x} & \text { if } G=S p \\ 1+\frac{\sin 2 \pi x}{2 \pi x} & \text { if } G=S O \text { (even) } \\ \delta_{0}+1-\frac{\sin 2 \pi x}{2 \pi x} & \text { if } G=S O \text { (odd). }\end{cases}
$$

As with the measures $\mu_{k}(\mathrm{GUE})$, the measures $\nu_{k}(G)$ may be expressed in terms of Fredholm determinants ([K-S1]), and this allows for their numerical calculation. The densities of $\nu_{1}(U), \nu_{1}(S p)$ and $\nu_{1}(S O($ even $))$ are displayed in Figure 5. Clearly, 
$\nu_{1}(S O($ odd $))=\delta_{0}$, and it turns out that $\nu_{2}(S O($ odd $))=\nu_{1}(S p)$. Note that $S p$ is unique in having the density of $\nu_{1}$ vanish (in fact to second order) at $s=0$. This shows that the eigenvalues of a typical $A$ in a large $\operatorname{USp}(2 N)$ are repelled by 1 .

We end this section by remarking that the same questions for the most reducible of the compact symmetric spaces, $T^{N}=U(1) \times U(1) \ldots \times U(1)$, have very different answers. Note that $T^{N}$ with the measure $\frac{d x_{1}}{2 \pi} \frac{d x_{2}}{2 \pi} \ldots \frac{d x_{N}}{2 \pi}$ corresponds to choosing $x_{1}, x_{2}, \ldots x_{N}$ independently at random (or if we think of these as matrices, then we are choosing a random diagonal matrix). The local spacing statistics for these have been much studied in the probability literature. It is well known [FE] that the local spacings for this model approximate a Poisson process as $N \rightarrow \infty$. The $k$-th consecutive spacing measures converge to $\mu_{k}(T)=s^{k-1} e^{-s} d s /(k-1)$ ! (note that $\mu_{1}$ has no repulsion at zero), while the limiting pair correlation $R_{2}(T)$ is simply the density $d x$ on $\mathbb{R}$.

\section{FUnCTION FIELDS}

One can get much insight into the source of the Montgomery Odlyzko Law by considering its function field analogue. Replace the field of rational numbers $\mathbb{Q}$ by a field $k$ which is a finite extension of the field $\mathbb{F}_{q}(t)$ of rational functions in $t$ with coefficients in $\mathbb{F}_{q}$, the finite field of $q$ elements. In analogy with the Riemann Zeta Function, Artin $[\mathrm{AR}]$ introduced a zeta function $\zeta(T, k)$. It is defined by the product over all places $v$ of $k$ (see [WE2] )

$$
\zeta(T, k)=\prod_{v}\left(1-T^{\operatorname{deg}(v)}\right)^{-1}
$$

One can also think of $\zeta(T, k)$ as the zeta function of a nonsingular curve $C$ over $\mathbb{F}_{q}$ whose field of functions is $k$. For example, let $C / \mathbb{F}_{q}$ be a plane curve given by an equation

$$
f\left(X_{1}, X_{2}, X_{3}\right)=0
$$

where $f$ is nonsingular and homogeneous of some degree and has coefficients in $\mathbb{F}_{q}$. For each $n \geq 1$ let $N_{n}$ be the number of projective solutions to (33) in $\mathbb{F}_{q^{n}}$. The zeta function of the field of functions $k$ of $C$ is the same as the zeta function of the curve $C$ over $\mathbb{F}_{q}$ which is defined as

$$
\zeta\left(T, C / \mathbb{F}_{q}\right)=\exp \left(\sum_{n=1}^{\infty} \frac{N_{n} T^{n}}{n}\right) .
$$

This geometric point of view is very powerful. For example, the Riemann-Roch Theorem on the curve $C$ plays the role of the Poisson summation formula [SCH] and shows that

$$
\zeta\left(T, C / \mathbb{F}_{q}\right)=\frac{P\left(T, C / \mathbb{F}_{q}\right)}{(1-T)(1-q T)}
$$

where $P \in \mathbb{Z}[T]$ is of degree $2 g, g$ being the genus of the curve $C$. It also gives the functional equation $P(T)=q^{g} T^{2 g} P(1 / q T)$. The Riemann-Hypothesis for these zeta functions, which was put forth and tested in many examples by Artin, asserts that all the zeroes lie on $|T|=1 / \sqrt{q}$. This was proven by Weil. By now there are several different proofs: Weil [WE3], [WE4], elementary proofs by Stepanov $[\mathrm{ST}]$ and Bombieri [BO], and proofs by Deligne [DE] which have the advantage of applying much more generally. One reason for being able to proceed in the function 
field setting is that one has a spectral interpretation of the zeroes of $\zeta\left(T, C / \mathbb{F}_{q}\right)$. The number $N_{n}$ is the number of fixed points of the $n$-th iterate of Frobenius (which is the operation of raising the co-ordinates of $\overline{\mathbb{F}}_{q}$-points on $C$ to the power $q$ ). Via a suitable Lefschetz trace formula this allows one to interpret the zeroes of $\zeta\left(T, C / \mathbb{F}_{q}\right)$ as the reciprocals of the eigenvalues of Frobenius acting on the first cohomology group (with $\ell$-adic coefficients) of the curve $C$.

Turning to the distribution of the zeroes of these zeta functions, we write them as

$$
\rho_{j}=e^{i \theta_{j}} / \sqrt{q}, j=1,2, \ldots, 2 g .
$$

Now order the $\theta$ 's as in (19) and form the local spacing measures as in $\left(19^{\prime}\right)$. We denote by $\mu_{k}\left(C / \mathbb{F}_{q}\right)$ the $k$-th consecutive spacing measure between its zeroes. For a fixed $\zeta\left(T, C / \mathbb{F}_{q}\right)$ there are $2 g(C)$ zeroes and so there cannot be any spacing law. We therefore let the genus go to infinity. However, this alone does not lead to a unique (or even the existence of a) limiting law. For example, consider the curves $C_{f}, f \geq 1$ given in affine form by $Y^{2}=X^{q}-X$ over the field $\mathbb{F}_{q}, q=p^{f}, p \neq 2$. Its genus is $(q-1) / 2$, and as explained in [K-S1] the consecutive spacing measure $\mu_{1}\left(C_{f} / \mathbb{F}_{q}\right)$ converges to a point mass $\delta_{0}$ at zero, as $f \rightarrow \infty$. In [K-S1] we therefore consider the "typical" curve of large genus over a large field $\mathbb{F}_{q}$. We show that as $q$ and $g$ go to infinity the local spacings between the zeroes follow the GUE model that is the Montgomery-Odlyzko Law is valid for these zetas. Precisely, if $\mathcal{M}_{g}\left(\mathbb{F}_{q}\right)$ denotes the (finite) set of isomorphism classes of curves of genus $g$ over $\mathbb{F}_{q}$ and $k \geq 1$, then

$$
\lim _{g \rightarrow \infty} \lim _{q \rightarrow \infty} \frac{1}{\# \mathcal{M}_{g}\left(\mathbb{F}_{q}\right)} \sum_{C \in \mathcal{M}_{g}\left(\mathbb{F}_{q}\right)} D\left(\mu_{k}\left(C / \mathbb{F}_{q}\right), \mu_{k}(\mathrm{GUE})\right)=0 .
$$

In particular, since $D \geq 0$ we have that for any $\epsilon>0$ and $q$ and $g$ large enough ( $g$ depending on $q$ )

$$
\#\left\{C \in \mathcal{M}_{g}\left(\mathbb{F}_{q}\right) \mid D\left(\mu_{k}\left(C / \mathbb{F}_{q}\right), \mu_{k}(\mathrm{GUE})\right)>\epsilon\right\} \leq \epsilon . \#\left\{C \in \mathcal{M}_{g}\left(\mathbb{F}_{q}\right)\right\} ;
$$

that is to say the zeta functions of almost all curves $C$ satisfy the MontgomeryOdlyzko Law as $q$ and $g$ go to infinity.

There are three key ingredients that go into the proof of (37). The first is the monodromy of the family $\mathcal{M}_{g}$. For technical reasons instead of the family of curves of genus $g$ one considers the family $\mathcal{M}_{g, 3 K}$ of curves of genus $g$ together with a basis of sections of $3 K, K$ being the canonical class of the curve. The monodromy representation of $\pi_{1}$ of $\mathcal{M}_{g, 3 K}$ on $H^{1}$ of a given curve has image whose Zariski closure (this being the monodromy of this family) is the full symplectic group $S p(2 g)$ [K-S1]. That this monodromy is symplectic in the first place is a consequence of it preserving the intersection pairing of cycles in the first homology group of a curve. Via this representation one can associate to each $C \in \mathcal{M}_{g}\left(\mathbb{F}_{q}\right)$ a unitarized Frobenius conjugacy class $\theta\left(C / \mathbb{F}_{q}\right)$ in $U S p(2 g)$ such that

$$
P\left(T, C / \mathbb{F}_{q}\right)=\operatorname{det}\left(1-\sqrt{q} T \theta\left(C / \mathbb{F}_{q}\right)\right) .
$$


The second ingredient is Deligne's main result [DE] which can be used to show (see $[\mathrm{K}-\mathrm{S} 1]$ ) that for any continuous class function $f$ on $U S p(2 g)$

$$
\lim _{q \rightarrow \infty} \frac{\sum_{C \in \mathcal{M}_{g}\left(\mathbb{F}_{q}\right)} \frac{f\left(\theta\left(C / \mathbb{F}_{q}\right)\right)}{\# \operatorname{Aut}\left(C / \mathbb{F}_{q}\right)}}{\sum_{C \in \mathcal{M}_{g}\left(\mathbb{F}_{q}\right)} \frac{1}{\# \operatorname{Aut}\left(C / \mathbb{F}_{q}\right)}}=\int_{U S p(2 g)} f(A) d A .
$$

The number of $C \in \mathcal{M}_{g}\left(\mathbb{F}_{q}\right)$ for which \# Aut $\left(C / \mathbb{F}_{q}\right) \geq 2$ can be shown to be of lower order as $q \rightarrow \infty$. Applying (40) with the continuous function $f(A)=$ $D\left(\mu_{k}(A), \mu_{k}(\mathrm{GUE})\right)$, together with the third ingredient - the Law of Large Numbers (22) of Section 2 (applied with $G(N)=U S p(2 N)$ ) - leads to (37).

One can prove similar results for other families of zeta or $L$-functions of curves or varieties $V$ over finite fields; see [K-S1]. The universal Montgomery-Odlyzko Law is valid at least if the monodromy of the family is big, that is, if it is (or is close to) any of the $G(N)$ 's of type II in Table 1 .

Thus in the function field, the source of the GUE phenomenon is clearly identified. It is the monodromy (or symmetry group) of the family and its scaling limit, combined with the universality of the spacing statistics for the type II symmetric spaces. The latter washes out the fine structure to the extent of not even betraying the specific symmetry type of the family. As was mentioned in Section 2 the measures $\nu_{k}$ are more sensitive, and we exploit this next.

\section{FAMILIES AND LOW LYING ZEROES}

The following table summarizes some known analogies between zeta functions over $\mathbb{Q}$ and function fields.

TABle 2

\begin{tabular}{|c|c|c|}
\hline & $A$ & $B$ \\
\hline $\begin{array}{l}1 \text { Type of } \\
\text { Zeta Function }\end{array}$ & $\begin{array}{l}\text { Number Field } \\
\zeta(s), L(s, \chi), L(s, f)\end{array}$ & $\begin{array}{l}\text { Function Field } \\
\zeta\left(T, C / \mathbb{F}_{q}\right), \zeta\left(T, V / \mathbb{F}_{q}\right), V \text { a variety. }\end{array}$ \\
\hline 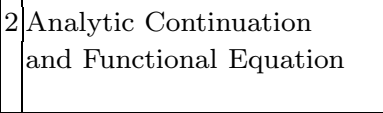 & $\begin{array}{l}\text { Established via Poisson } \\
\text { Summation or Automorphic } \\
\text { Properties of } f \text {. }\end{array}$ & $\begin{array}{l}\text { Established using Riemann-Roch } \\
\text { and in general Étale Cohomology. }\end{array}$ \\
\hline $\begin{array}{l}3 \text { Spectral Interpretation } \\
\text { of Zeroes }\end{array}$ & ? & $\begin{array}{l}\text { Known via the Action of Frobenius } \\
\text { on Cohomology. }\end{array}$ \\
\hline $4 \mathrm{RH}$ & Expected & Proven, Weil, Deligne. \\
\hline 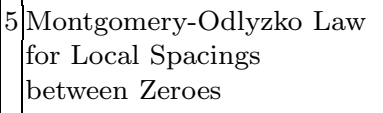 & Expected & Valid for almost all curves. \\
\hline 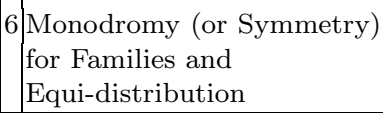 & $?$ & $\begin{array}{l}\text { Known via the representation } \\
\text { of } \pi_{1} \text { of the Family } \\
\text { on Cohomology Groups. }\end{array}$ \\
\hline
\end{tabular}


We view the entry 5-A and the evidence towards it described in Sections 1 and 3 as strongly suggesting an entry of "expected" in 3 -A. ${ }^{1}$ Also worth noting is that Deligne's first proof of 4-B is based on monodromy for a family. For example, to establish RH for $\zeta(T, V), V$ a smooth hyper-surface in $\mathbb{P}^{N}$, he proceeds by putting $V$ in a family $V_{t}$ (for an exposition see [KA1]). RH is established for all $V_{t}$ 's at once by exploiting the monodromy of the family which glues the $V_{t}$ 's together. Thus, a positive entry in 6-A is most desirable. This is the topic of this section.

We begin with the function field where the notion of a family has a precise meaning. Consider the set of all quadratic extensions of $k=\mathbb{F}_{q}(t)$. We may realize these as follows: Let $H_{n}\left(\mathbb{F}_{q}\right)$ be the set of all monic square-free polynomials of degree $n$ with coefficients in $\mathbb{F}_{q}$. The quadratic extensions are $k_{\Delta}=k(\sqrt{\Delta})$ with $\Delta \in H_{n}\left(\mathbb{F}_{q}\right)$. For each $n$, these function fields correspond to hyper-elliptic curves, $Y^{2}=\Delta(\mathrm{X}), \Delta \in H_{n}$ and form a family of curves. The genus $g=g(n)$ of $k_{\Delta}$ satisfies $n=2 g+2$ if $n$ is even and $n=2 g+1$ if $n$ is odd. Thus $\zeta\left(T, k_{\Delta}\right)$, which may be written as $L\left(T, \chi_{\Delta}\right) /(1-T)(1-q T)$ with $\chi_{\Delta}$ the corresponding quadratic character, has $2 g(n)$ zeroes with angles $\theta_{1}(\Delta), \theta_{2}(\Delta), \ldots, \theta_{2 g}(\Delta)$ which we normalize and order as in (36) and (19). For $j \geq 1$ fixed we examine the distribution of $\theta_{j}(\Delta)$ as $\Delta$ varies over $H_{n}\left(\mathbb{F}_{q}\right), n \rightarrow \infty$. This amounts to studying the distribution of the zeroes near the central point. Using the equi-distribution techniques described in Section 3 together with the $\nu$ scaling limits (30) for $U S p(2 g)$ (the monodromy of this hyper-elliptic family again being the full $S p(2 g)$ ), one shows (see [K-S1]) that for $f \in C_{0}\left(\mathbb{R}_{\geq 0}\right)$ a test function

$$
\lim _{n \rightarrow \infty} \lim _{q \rightarrow \infty} \frac{1}{\# H_{n}\left(\mathbb{F}_{q}\right)} \sum_{\Delta \in H_{n}\left(\mathbb{F}_{q}\right)} f\left(\frac{\theta_{j}(\Delta) 2 g}{2 \pi}\right)=\int_{0}^{\infty} f(x) d \nu_{j}(S p)(x) .
$$

We conjecture (though the techniques leading to (41) offer nothing in the way of a proof) that (41) holds without taking the inner $q$ limit, that is

$$
\lim _{n \rightarrow \infty} \frac{1}{\# H_{n}\left(\mathbb{F}_{q}\right)} \sum_{\Delta \in H_{n}\left(\mathbb{F}_{q}\right)} f\left(\frac{\theta_{j}(\Delta) g}{\pi}\right)=\int_{0}^{\infty} f(x) d \nu_{j}(S p)(x) .
$$

Equations (41) and (42) assert that the distributions of the zeroes near $T=1 / \sqrt{q}$ (the central or symmetry point) of the quadratic extensions $k_{\Delta}$ of $k=\mathbb{F}_{q}(t)$ follow the symplectic scaling limit laws.

We give a second example in the function field of a family for which the distribution of the zeroes near the central point is dictated by, and in turn reveals, the symmetry group of the family. Let $E / k$ be an elliptic curve with non-constant $j$-invariant ([SI1]). For each place $v$ of $k$ (that is each irreducible polynomial $p(t) \in \mathbb{F}_{q}[t]$ or the place at infinity - "degree") the residue field $k_{v}$ is a finite extension of $\mathbb{F}_{q}$ of degree $d_{v}$. Let $E_{v}$ be the curve $E \otimes k_{v}$. At a place at which $E$ has good reduction, write

$$
\left|E_{v}\right|=\left|k_{v}\right|+1-\alpha_{v}-\beta_{v} \quad \text { with } \quad \alpha_{v} \beta_{v}=\left|k_{v}\right| .
$$

\footnotetext{
${ }^{1} \mathrm{An}$ interesting possibility for a spectral interpretation of the zeroes has been suggested by Connes $[\mathrm{CO}]$.
} 
The $L$-function of $E / k$ is defined to be

$$
L(T, E)=\prod_{v}\left(1-\alpha_{v} T^{d_{v}}\right)^{-1}\left(1-\beta_{v} T^{d_{v}}\right)^{-1}
$$

(for the places $v$ of $k$ at which $E$ does not have good reduction, the local factor is described in [SI1]). $L(T, E)$ satisfies a functional equation and Riemann Hypothesis [TA], [DE].

For example, consider the Legendre curve

$$
E: Y^{2}=X(X-t)(X-1) \text {. }
$$

For $\Delta \in \tilde{H}_{n}\left(\mathbb{F}_{q}\right)$, the set of $\Delta \in H_{n}\left(\mathbb{F}_{q}\right)$ with no zeroes in $\{0,1\}$, the twist of $E$ by $\chi_{\Delta}$ has $L$-function

$$
L\left(T, E \otimes \chi_{\Delta}\right)=\prod_{v}\left(1-\alpha_{v} \chi_{\Delta}(v) T^{d_{v}}\right)^{-1}\left(1-\beta_{v} \chi_{\Delta}(v) T^{d_{v}}\right)^{-1},
$$

and its degree is $N$ where $N$ is $2 n$ if $n$ is even and $2 n-1$ if $n$ is odd. The zeroes of $L\left(T, E \otimes \chi_{\Delta}\right)$ are of modulus $1 / q$, and we write them as $q^{-1} e^{i \theta_{1}}, q^{-1} e^{i \theta_{2}}, \ldots, q^{-1} e^{i \theta_{N}}$ with

$$
0 \leq \theta_{1}\left(E \otimes \chi_{\Delta}\right) \leq \theta_{2}\left(E \otimes \chi_{D}\right) \ldots \leq \theta_{N}\left(E \otimes \chi_{\Delta}\right)<2 \pi .
$$

Denote by $\epsilon\left(E \otimes \chi_{D}\right)(= \pm 1)$ the sign of the functional equation. Each of the two values is shared roughly equally by the $\Delta$ 's in $\tilde{H}_{n}\left(\mathbb{F}_{q}\right)$ as $n \rightarrow \infty$. Let $\tilde{H}_{n}^{ \pm}\left(\mathbb{F}_{q}\right)$ denote those $\Delta \in \tilde{H}_{n}\left(\mathbb{F}_{q}\right)$ with $\epsilon$ equal to +1 and -1 respectively. As with the last example one can (see [KA2]) compute the monodromy of this family $L\left(T, E \otimes \chi_{\Delta}\right)$, $\Delta \in \tilde{H}_{n}\left(\mathbb{F}_{q}\right)$. It is equal to $O(N) .^{2}$ As above, the equi-distribution techniques together with (30) with $G=O$ lead to: Fix $j \geq 1$ and $f \in C_{0}\left(\mathbb{R}_{\geq 0}\right)$; then (see [K-S1])

$$
\lim _{n \rightarrow \infty} \lim _{q \rightarrow \infty} \frac{1}{\# \tilde{H}_{n}^{+}\left(\mathbb{F}_{q}\right)} \sum_{\Delta \in \tilde{H}_{n}^{+}\left(\mathbb{F}_{q}\right)} f\left(\frac{N \theta_{j}\left(E \otimes \chi_{\Delta}\right)}{2 \pi}\right)=\int_{0}^{\infty} f(x) d \nu_{j}(S O(\text { even }))(x)
$$

and

$$
\lim _{n \rightarrow \infty} \lim _{q \rightarrow \infty} \frac{1}{\# \tilde{H}_{n}^{-}\left(\mathbb{F}_{q}\right)} \sum_{\Delta \in \tilde{H}_{n}^{-}\left(\mathbb{F}_{q}\right)} f\left(\frac{N \theta_{j}\left(E \otimes \chi_{\Delta}\right)}{2 \pi}\right)=\int_{0}^{\infty} f(x) d \nu_{j}(S O(\text { odd }))(x) .
$$

So this is a family with an orthogonal symmetry and a corresponding distribution of zeroes near the central point. As in the first example we conjecture that (48) and (49) hold without the inner $q$ limit - that is they hold for a fixed $q$.

In principle in this function field setting, as long as one can compute the monodromy of the family and its scaling limits, as is done in the above examples, one obtains the scaled spacing distribution of zeroes near the central point.

We turn to the rational number setting. The analogues of the two families considered above are clear enough. The first consists of the Dirichlet $L$-functions

\footnotetext{
${ }^{2}$ The reason for this difference between the families $L\left(s, \chi_{\Delta}\right)$ and $L\left(s, E \otimes \chi_{\Delta}\right)$ is that the Frobenius acts on $H^{1}$ of a curve with coefficients which are orthogonally self dual in the first case and symplectically self dual in the second. By standard properties of cup-product, the autoduality on such $H^{1}$ is reversed.
} 
$L(s, \chi)$ with $\chi^{2}=1$. The second of $L(s, E \otimes \chi)$ where $E / \mathbb{Q}$ is an elliptic curve and $\chi$ a quadratic character as in the first family. The corresponding question concerns the distribution of the zeroes near $s=1 / 2$ (we always normalize the $L$-functions to have functional equation $s \rightarrow 1-s$ so that $1 / 2$ is the central point) of these $L$ functions. We will examine a number of different families $\mathcal{F}$. To each automorphic form $f \in \mathcal{F}$ let $c_{f}$ be its conductor (see the line above (12)). Assume further that the sets $\mathcal{F}_{\mathrm{X}}=\left\{f \in \mathcal{F} \mid c_{f} \leq \mathrm{X}\right\}$ are finite and that the asymptotics $\# \mathcal{F}_{\mathrm{X}}$, as $\mathrm{X}$ goes to infinity, can be determined. Write the nontrivial zeroes of $L(s, f)$ as

$$
\frac{1}{2}+i \gamma_{f}
$$

and assuming $\mathrm{RH}$ order these

$$
\cdots \leq \gamma_{f}^{(-2)} \leq \gamma_{f}^{(-1)} \leq 0 \leq \gamma_{f}^{(1)} \leq \gamma_{f}^{(2)} \leq \ldots .
$$

For $j \geq 1$ consider the distribution of the numbers

$$
\frac{\gamma_{f}^{(j)} \log c_{f}}{2 \pi}
$$

as $f$ varies over $\mathcal{F}_{\mathrm{X}}, \mathrm{X} \rightarrow \infty$. That is, we study the distribution of the $j$-th lowest zero (that the normalization (52) is appropriate will become clear from the results below). Form the analogues of the measures $\nu_{k}$ (see (27)) and the density $\Delta$ and $W((28),(29))$

$$
\nu_{j}(\mathrm{X}, \mathcal{F})[a, b]=\frac{\#\left\{f \in \mathcal{F}_{\mathrm{X}} \mid \frac{\gamma_{f}^{(j)} \log c_{f}}{2 \pi} \in[a, b]\right\}}{\# \mathcal{F}_{\mathrm{X}}} .
$$

For $\phi \in \mathcal{S}(\mathbb{R})$ a test function, set

$$
\Delta(f, \phi)=\sum_{\gamma_{f}} \phi\left(\frac{\gamma_{f} \log c_{f}}{2 \pi}\right)
$$

and

$$
W(\mathrm{X}, \mathcal{F}, \phi)=\frac{1}{\# \mathcal{F}_{\mathrm{X}}} \sum_{c_{f} \leq \mathrm{X}} \Delta(f, \phi) .
$$

One can also form several variable joint densities (see [K-S1]), and these determine the $\nu_{j}$ 's as well as all the local scaled distributions of zeroes near $s=1 / 2$. By analogy with the function field we might expect that $\nu_{j}(\mathrm{X}, \mathcal{F})$ and $W(\mathrm{X}, \mathcal{F}, \phi)$ converge to $\nu_{j}(\mathcal{F})$ and $\int_{-\infty}^{\infty} \phi(x) w(\mathcal{F})(x) d x$, where $\nu_{j}(\mathcal{F})$ and $w(\mathcal{F})$ correspond to the symmetry type " $G(\mathcal{F})$ ". If the family $\mathcal{F}$ has a function field analogue, then $G(\mathcal{F})$ and the corresponding $\nu_{j}(\mathcal{F}), w(\mathcal{F})$ can be predicted by the analysis described in the two examples above. This idea has been carried out and tested analytically and numerically for various families, some of which are as follows (for the rest of this section we assume $\mathrm{RH}$ for all $L(s, f)$ 's):

I. The family $\mathcal{F}$ of Dirichlet $L$-functions $L(s, \chi), \chi$ (primitive) of conductor $c_{\chi}=q$ and $\chi$ quadratic (i.e. $\left.\chi^{2}=1\right) . L(s, \chi)$ is self-dual (that is its functional equation is back into itself) and the sign $\epsilon_{\chi}$ is equal to 1 for all $\chi$.

(a) The discussion of the function field analogue suggests that $G(\mathcal{F})=S p$. 


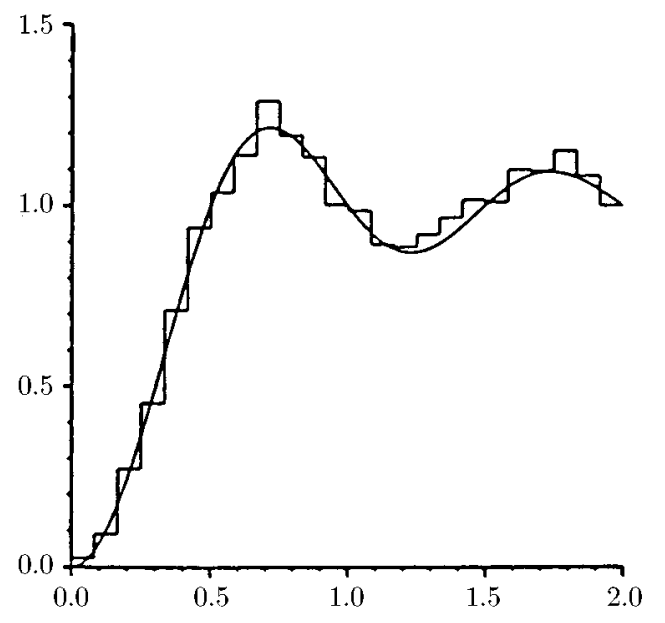

Figure 6. 1 level density for $L\left(s, \chi_{d}\right), 10^{12}<|d|<10^{12}+$ 200000,7243 d's, mean of 1 st zero above 0 equals 0.8268 , renormalized to have mean 0.78 .

(b) $[\mathrm{K}-\mathrm{S} 2]$ (see also Ozluk-Snyder $[\mathrm{O}-\mathrm{S}]$ )

$$
W(\mathrm{X}, \mathcal{F}, \phi) \rightarrow \int_{-\infty}^{\infty} \phi(x) w(S p)(x) d x \quad \text { as } X \rightarrow \infty
$$

for any $\phi \in \mathcal{S}(\mathbb{R})$ whose Fourier transform is supported in $(-2,2)$. The density $w(S p)(x)$ is given in $\left(31^{\prime}\right)$.

(c) In his recent thesis [RUB] Rubinstein establishes that the $n \geq 2$ joint densities converge to the $S p$ densities (see [K-S1]) for test functions $\phi\left(x_{1}, \ldots, x_{n}\right)$ whose Fourier transforms are supported in a small neighborhood of 0 .

(d) Numerical experiments, Rubinstein [RUB] for $q$ of size $10^{12}$ give an excellent fit of the $\nu_{j}$ 's and $W$ with the $S p$ predictions. For example, the density of zeroes (scaled) versus $w(S p)$ is given in Figure 6 . The distribution of the lowest zero $\nu_{1}(\mathrm{X}, \mathcal{F})$ for $q \sim 10^{12}$ is displayed in Figure 7. It is compared with $\nu_{1}(S p)$. Note that in the analysis I(b) above, the convergence to the limit is at a speed of $1 / \log q$ and moreover there is a term of one sign which shifts the answer by this amount. While this term disappears in the limit $q \rightarrow \infty$, it does affect the mean numerically, and the data displayed incorporates a re-normalization taking this into account. According to the $S p$ predictions, the mean value of $\frac{\gamma_{\chi} \log q_{\chi}}{2 \pi}$ should be the mean of $\nu_{1}(S p)$ which is $0.7827 \ldots$ The numerics confirm this though the convergence is slow (like $1 / \log q)$ and the approach is from above.

(e) The first person to compute numerically the zeroes of $L(s, \chi)$ in this family appears to be Hazelgrave. He found that the zeroes "repel" the point $s=1 / 2$, and this is referred to as the Hazelgrave phenomenon. ${ }^{3}$

\footnotetext{
${ }^{3}$ One can carry out all of the above for $L(s, \chi), \chi^{3} \equiv 1$ (or any other order bigger than 2 ). One finds a unitary symmetry and the zeroes do not repel $s=1 / 2$.
} 


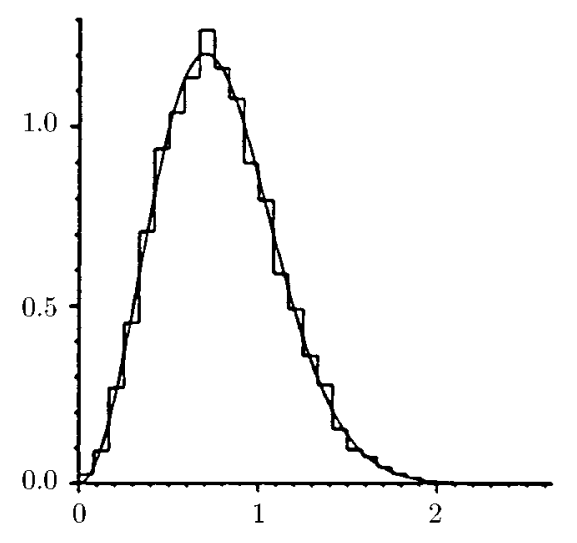

Figure 7. 1st zero above 0 for $L\left(s, \chi_{d}\right), 10^{12}<|d|<10^{12}+$ 200000,7243 d's, mean of 1 st zero above 0 equals 0.8268 , renormalized to have mean 0.7827 .

As remarked in Section 2 the density $\nu_{1}(S p)$ vanishes to second order at $s=0$. Thus, the Hazelgrave phenomenon is a manifestation of the symplectic symmetry!

II. The family $\Delta \otimes \chi$ where $\Delta$ is the weight 12-cusp form in (15) and $\chi$ runs over the quadratic characters. The conductor $c_{\Delta \otimes \chi}$ is $q^{2}$ and $\epsilon_{\Delta \otimes \chi}$ can be both +1 and -1 . $L(s, \Delta \otimes \chi)$ is self-dual.

(a) From function field considerations we expect $G(\mathcal{F})=O$ with the refinement that the sub-family $\mathcal{F}^{+}$with $\epsilon=1$ has an $S O$ (even) symmetry and $\mathcal{F}^{-}$with $\epsilon=-1$ an $S O$ (odd) symmetry.

(b) [K-S2] For this family or more generally with any $G L_{2}$ cusp form $f$ (with trivial central character) replacing $\Delta$, we have

$$
W\left(\mathrm{X}, \mathcal{F}^{+}, \phi\right) \rightarrow \int_{-\infty}^{\infty} \phi(x) w(S O(\text { even }))(x) d x
$$

and

$$
W\left(\mathrm{X}, \mathcal{F}^{-}, \phi\right) \rightarrow \int_{-\infty}^{\infty} \phi(x) w(S O(\text { odd }))(x) d x
$$

for any $\phi \in \mathcal{S}(\mathbb{R})$ whose Fourier transform is supported in $(-1,1)$. The densities $w(S O)(x)$ are given in $\left(31^{\prime}\right)$. The last applies in particular to $L(s, E \otimes \chi)$ with $E / \mathbb{Q}$ an elliptic curve, which of course is consistent with the function field example mentioned at the beginning of this section.

(c) Rubinstein [RUB] has shown that the $n \geq 2$ level densities converge to the orthogonal densities for a $\phi\left(x_{1}, \ldots, x_{n}\right)$ with $\hat{\phi}\left(\xi_{1}, \ldots, \xi_{n}\right)$ supported in a small neighborhood of 0 .

(d) Numerical experiments (Rubinstein [RUB] ) for $q$ of size about 500,000 give an excellent fit with the orthogonal predictions. In Figure 8, the density of $\mathcal{F}^{+}$is plotted against $w(S O$ (even)), and in Figure 9 the density of $\mathcal{F}^{-}$against $w(S O$ (odd)) (for the latter there is always a zero at $s=1 / 2$ and a $\delta_{0}$ in the density; these are suppressed). In Figure 


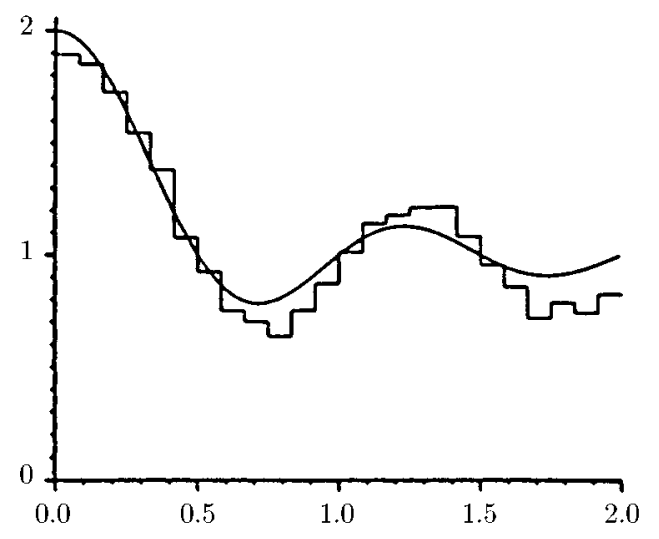

FiguRE 8. 1-level density for $L_{\tau}\left(s, \chi_{d}\right.$ even $), 350000<|d|<$ $650000,11464 d$ 's, mean of 1 st zero above 0 equals 0.2926 , renormalized to have mean 0.3214 .

$10, \nu_{1}\left(\mathcal{F}^{+}\right)$is displayed against $\nu_{1}(S O($ even $))$, and in Figure $11 \nu_{2}\left(\mathcal{F}^{-}\right)$ against $\nu_{2}(S O$ (odd)).

III. The family $H(N)$ of holomorphic Hecke-eigen forms of weight 2 for $\Gamma_{0}(N)$ (as in (18)). For simplicity we assume that $N$ is prime. $L(s, f)$ has conductor $c_{f}=N$ and is self-dual. As in the last family approximately half of the $f$ 's have $\epsilon_{f}=1$, with the remaining half having $\epsilon_{f}=-1$. Let $H^{+}(N)$ and $H^{-}(N)$ denote the corresponding sets. We have that $\#\left(H^{+}(N)\right) \sim$ $\#\left(H^{-}(N)\right) \sim N / 24$ as $N \rightarrow \infty$.

(a) The expected symmetry $G(\mathcal{F})$ is $O$, though as yet we have not understood the function field analogue.

(b) (See Iwaniec-Luo-Sarnak [I-L-S]):

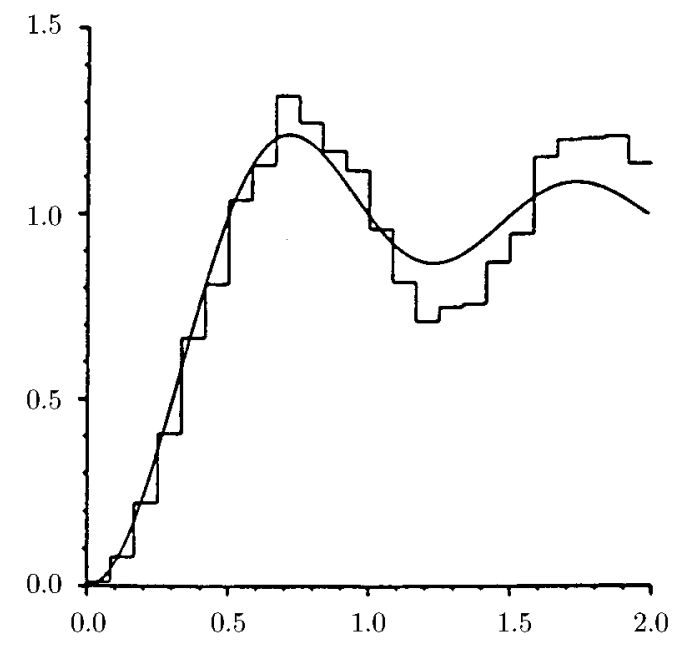

Figure 9. 1-level density for $L_{\tau}\left(s, \chi_{d}\right.$ odd) $350000<|d|<$ $650000,11390 d$ 's, mean of 1st zero above 0 equals 0.7186 , renormalized to have mean 0.7827 . 


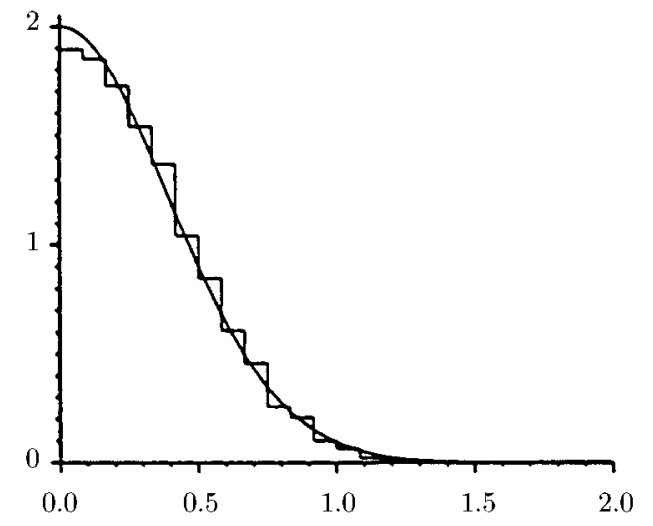

Figure 10. 1st zero above 0 for $L_{\tau}\left(s, \chi_{d}\right.$ even) $350000<|d|<$ $650000,11464 d$ 's, mean of 1 st zero above 0 equals 0.2926 , renormalized to have mean 0.3214 .

$$
\begin{aligned}
& \text { As } N \rightarrow \infty \\
& \frac{1}{\#\left(H^{+}(N)\right)} \sum_{f \in H^{+}(N)} \Delta(\phi, f) \rightarrow \int_{-\infty}^{\infty} \phi(x) w(S O(\text { even }))(x) d x \\
& \frac{1}{\#\left(H^{-}(N)\right)} \sum_{f \in H^{-}(N)} \Delta(\phi, f) \rightarrow \int_{-\infty}^{\infty} \phi(x) w(S O(\text { odd }))(x) d x
\end{aligned}
$$

for any $\phi \in \mathcal{S}(\mathbb{R})$ with support $\hat{\phi} \subset(-2,2)$.

IV. The family of symmetric-square $L$-functions (see [SH1] ) $L\left(s, s^{2} m^{2} f\right.$ ) where $f$ is a Hecke eigenform of even integral weight $k$ on $\mathbb{H}$ for $\Gamma=S L_{2}(\mathbb{Z})$ (as in (15) above). The dimension of this space of cusp forms is $\sim \frac{k}{12}$ as $k \rightarrow \infty$. The appropriate (analytic) analogue of the conductor of $c_{s y m^{2} f}$ of $L\left(s, s y m^{2} f\right)$ is $k^{2}$. For this family the averaging is over all such $s y m^{2} f$

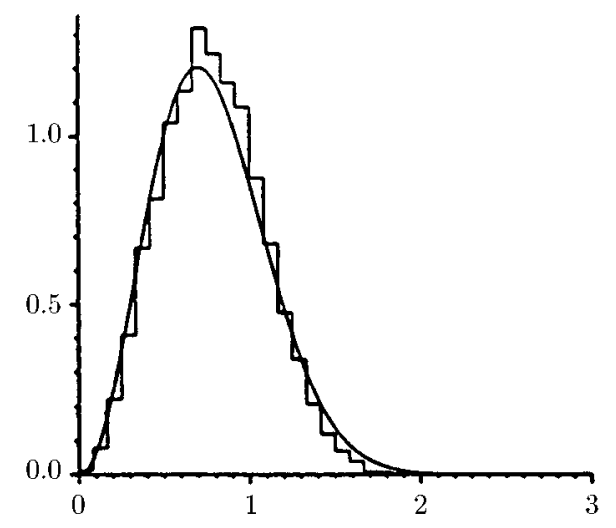

FiguRe 11. 1st zero above 0 for $L_{\tau}\left(s, \chi_{d}\right.$ odd) $350000<|d|<$ $650000,11390 d$ 's, mean of 1 st zero above 0 equals 0.7186 , renormalized to have mean 0.7827 . 
with $c_{s y m^{2} f} \leq \mathrm{X} . L\left(s, s y m^{2} f\right)$ is an Euler-product of degree three, and by a theorem of Gelbart and Jacquet [G-J] these are $L$-functions of self-dual cusp forms on $G L_{3}$. The sign of the functional equation $\epsilon_{s y m^{2} f}$ is equal to 1.

(a) Being self-dual forms on $G L_{3}$ we expect $G(\mathcal{F})=S p$.

(b) In [I-L-S] it is proven that

$$
W(\mathrm{X}, \mathcal{F}, \phi) \rightarrow \int_{-\infty}^{\infty} \phi(x) w(S p)(x) d x
$$

as $\mathrm{X} \rightarrow \infty$, for any $\phi \in \mathcal{S}(\mathbb{R})$ with support $\hat{\phi} \subset\left(-\frac{4}{3}, \frac{4}{3}\right)$.

Remarks. 1. All of these results confirm, to the extent that they apply, the predictions of the $G(\mathcal{F})$ symmetry. We call the conjecture that the sums $W(\mathrm{X}, \mathcal{F}, \phi)$ converge to the claimed density without any restrictions on $\hat{\phi}$, the Density Conjecture for $\mathcal{F}$.

2. The proofs of the results about densities all proceed by expressing $\Delta(f, \phi)$ via the explicit formula, in terms of sums involving the Hecke eigenvalues of $f$. The method used for averaging such quantities over $f \in \mathcal{F}$ for the families III and IV draws heavily on the tools developed in Iwaniec-Sarnak [I-S] (see also Section 5).

3 . With the exception of the family II, all of the results allow for the support of $\hat{\phi}$ to be larger than $[-1,1]$. This is rather significant since $\widehat{w(S p)}(\xi)$, $w(\widehat{S O)(\text { even })}(\xi)$ and $w(\widehat{S O)(\mathrm{odd}})(\xi)$ are all discontinuous at $\xi= \pm 1$. This signals that new non-diagonal terms enter as main terms as soon as the support of $\hat{\phi}$ is larger than $[-1,1]$. Thus, what is shown here goes beyond anything that has been established for the correlations of the high zeroes of $\zeta(s)$, as discussed in Section 1. For the families III and IV these new terms arise out of a far reaching analysis with Kloosterman sums (see also [D-F-I] for related analyses). That these fundamentally new non-diagonal contributions yield precisely the conjectured $G(\mathcal{F})$ answers is very pleasing evidence for this symmetry picture, at least for these families.

4. Other families for which similar results have been derived are: The family $L(s, f), f$ varying over holomorphic cusp forms of weight $k$ for $S L_{2}(\mathbb{Z})$ as $k \rightarrow \infty$, with symmetry $G(\mathcal{F})=O$ [I-L-S]. The family $L\left(s, f \otimes \chi_{D}\right)$ as in II above but where $f$ is now any fixed self-dual cusp form on $G L_{m} / \mathbb{Q}$, whose symmetry is $S p$ or $O$ according to whether $L\left(s, s y m^{2} f\right)$ does or does not have a pole at $s=1$ [K-S2]. The family $L(s, \chi) \chi^{n}=1, n \geq 3$. These $L$-functions are not self-dual and the symmetry is $U^{n}$ - the subgroup of the unitary group whose elements have their determinant an $n$-th root of 1 [KA3].

\section{Applications}

The interest in the zeroes of $L$-functions lies in their fundamental influence on arithmetical problems. In particular, the question of vanishing of an $L$-function at a special point on the critical line arises in the Birch and Swinnerton-Dyer Conjecture [B-S] (which for certain $f$ relates the vanishing of $L(s, f)$ and its derivatives to ranks of elliptic curves and abelian varieties (see below)), in the Shimura correspondence [SH2] and in spectral deformation theory (see Phillips-Sarnak [P-S]). The distribution of zeroes for a family $L(s, f)$ near $s=1 / 2$ as discussed in Section 4 has immediate applications to vanishing at that point. By varying the test function $\phi$ 
in the Density Conjecture for any of the above families $\mathcal{F}$, one is led to (assuming the Density Conjecture):

$$
\lim _{\mathrm{X} \rightarrow \infty} \frac{\#\left\{f \in \mathcal{F}_{\mathbf{X}} \mid \epsilon_{f}=1, L\left(\frac{1}{2}, f\right) \neq 0\right\}}{\#\left\{f \in \mathcal{F}_{\mathbf{X}} \mid \epsilon_{f}=1\right\}}=1
$$

and

$$
\lim _{\mathrm{X} \rightarrow \infty} \frac{\#\left\{f \in \mathcal{F}_{\mathrm{X}} \mid \epsilon_{f}=-1, L^{\prime}\left(\frac{1}{2}, f\right) \neq 0\right\}}{\#\left\{f \in \mathcal{F}_{\mathrm{X}} \mid \epsilon_{f}=-1\right\}}=1 .
$$

The results of Section 4 are approximations to the Density Conjecture and give corresponding approximations to (56) and (57). We illustrate this in the cases of families II(b) and III.

Let $E / \mathbb{Q}$ be an elliptic curve which is given in the form

$$
E: Y^{2}=\mathrm{X}^{3}+A \mathrm{X}+B .
$$

The twist of $E$ by a square free integer $D$ is the curve

$$
E_{D}: D Y^{2}=\mathrm{X}^{3}+A \mathrm{X}+B .
$$

We assume that $E$ is modular so that the $L$-function $L\left(s, E_{D}\right)$, which equals $L\left(s, E \otimes \chi_{D}\right)$, is also modular. The Birch and Swinnerton-Dyer Conjecture assert that the order of vanishing of $L\left(s, E_{D}\right)$ at $s=1 / 2$ is equal to the rank of the group of $\mathbb{Q}$-rational points on $E_{D}$. Kolyvagin $[\mathrm{K}-\mathrm{L}]$ has shown that if $L\left(\frac{1}{2}, E_{D}\right) \neq 0$, then $\operatorname{rank}\left(E_{D}\right)=0$. This together with the Density-Conjecture via (56) implies the following conjectures of Goldfeld [GO]:

- The rank of $E_{D}$ is zero for $100 \%$ of the $D$ 's with $|D| \leq \mathrm{X}, \epsilon_{E \otimes \chi_{D}}=1$, as $\mathrm{X} \rightarrow \infty$.

- Assuming further the Birch and Swinnerton-Dyer Conjecture and applying (57) show that the rank of $E_{D}$ is equal to one for $100 \%$ of the $D$ 's with $|D| \leq \mathrm{X}, \epsilon_{E \otimes \chi_{D}}=-1$, as $\mathrm{X} \rightarrow \infty$.

The approximation II(b) of Section 4 implies (assuming RH for the $L\left(s, E_{D}\right)$ 's) that rank $\left(E_{D}\right)=0$ for at least $25 \%$ of the $D$ 's with $\epsilon_{E_{D}}=1$. This result is due to Brumer and Heath-Brown [B-HB]. A challenging problem, which is as yet at the border of known techniques (see [P-P]), is to give an unconditional proof that rank $E_{D}=0$ for a positive proportion of $|D| \leq \mathrm{X}$, as $\mathrm{X} \rightarrow \infty$. (For certain curves $E$ such a result is known by algebraic methods [HB], [WO]). We note that for this family $L\left(s, E \otimes \chi_{D}\right)$ (as well as for some others below), unlike the case of $L\left(s, \Delta \otimes \chi_{D}\right)$ discussed in II, there are many $D$, with $\epsilon_{E \otimes \chi_{D}}=1$ and $L\left(\frac{1}{2}, E \otimes \chi_{D}\right)=0$. By choosing $\mathrm{X}$ and $Y$ first and then $D$ in (59), Gouvea and Mazur [GO-MA] show that there are at least $\mathrm{X}^{1 / 2}, D$ 's with $|D| \leq \mathrm{X} \epsilon_{D}=1$ and $L\left(\frac{1}{2}, E \otimes \chi_{D}\right)=0$, as $\mathrm{X} \rightarrow \infty$. This certainly affects the numerics for the distribution of low-lying zeroes for this family when $\mathrm{X}$ is of moderate size.

We turn to the family in III. By choosing $\phi \in \mathcal{S}(\mathbb{R}), \phi(0)=1, \phi \geq 0$, support $\hat{\phi} \subset$ $(-2,2)$ and for which $\int_{-\infty}^{\infty} \phi(x) w(\mathcal{F}, x) d x$ is minimized (see [I-L-S]), we conclude from the results in III (which we recall assume RH for $L(s, f)$ ) that for $N$ prime and large enough

$$
\frac{\#\left\{f \in H^{+}(N) \mid L\left(\frac{1}{2}, f\right) \neq 0\right\}}{\# H^{+}(N)}>\frac{9}{16}
$$




$$
\frac{\#\left\{f \in H^{-}(N) \mid L^{\prime}\left(\frac{1}{2}, f\right) \neq 0\right\}}{\# H^{-}(N)}>\frac{15}{16}
$$

and

$$
\frac{\# H_{2}(N)}{2}+\circ\left(\# H_{2}(N)\right) \leq \sum_{f \in H_{2}(N)} \text { ord }\left(\frac{1}{2}, L(s, f)\right)<\frac{99}{100}(\# H(N))
$$

where $\operatorname{ord}\left(s_{0}, L(s, f)\right)$ is the order of vanishing of $L(s, f)$ at $s=s_{0}$. Note that $\# H(N) \sim \frac{N}{12}$ and as Murty [MU] points out (and this does not assume $\mathrm{RH}$ ) $\# H^{ \pm}(N) \sim \# \frac{H(N)}{2}$. Thus, the lower bound in (62) is clear. Concerning the upper bound in (62), Brumer [BR] established such a result with 99/100 replaced by $3 / 2$. One can reduce this $3 / 2$ to 1 without appealing to the "off-diagonal" analysis which leads to III. However, to get anything below 1 in (62) already relies on the extended ranges in III. A similar remark applies to (60), the off-diagonal analysis allowing for the lower bound which exceeds $50 \%$. This is significant as will become clear from what follows.

One can apply (60), (61) and (62) to estimate the rank of the Jacobian $J_{0}(N) / \mathbb{Q}$ of the curves $X_{0}(N)$ (which analytically is $\Gamma_{0}(N) \backslash \mathbb{H}$ ) by combining those results with known partial results towards the Birch and Swinnerton-Dyer Conjectures (Kolyvagin [K-L] and Gross-Zagier [G-Z]). Let $M_{0}(N) / \mathbb{Q}$ be the quotient of $J_{0}(N)$ considered by Merel [MER]. It corresponds to those $f \in H^{+}(N)$ for which $L\left(\frac{1}{2}, f\right) \neq$ 0 , and it is no doubt the largest quotient of $J_{0}(N)$ of rank zero. It is of great interest to know its size ([MA],[MER]). Brumer $[\mathrm{BR}]$ has computed these for $N \leq 10^{4}$, and based on his findings, he conjectures that

$$
\begin{aligned}
& \lim _{N \rightarrow \infty} \frac{\operatorname{dim} M_{0}(N)}{\# H^{+}(N)}=1 \\
& \lim _{N \rightarrow \infty} \frac{\operatorname{rank} J_{0}(N)}{\operatorname{dim} J_{0}(N)}=\frac{1}{2} .
\end{aligned}
$$

Note that Density Conjectures for this family via (56) and (57) and [K-L] and [G-Z] imply these conjectures of Brumer. In the same way (60),(61) imply (still assuming $\mathrm{RH}$ for $L(s, f))$ that for $N$ large

$$
\operatorname{dim} M_{0}(N)>\frac{9}{16} \# H^{+}(N)
$$

and

$$
\operatorname{rank} J_{0}(N)>\frac{15}{32} \operatorname{dim} J_{0}(N) .
$$

Moreover, if one further assumes the Birch and Swinnerton-Dyer Conjecture then (62) shows that for $N$ large

$$
\frac{\operatorname{dim} J_{0}(N)}{2}+\circ(N) \leq \operatorname{rank} J_{0}(N)<\frac{99}{100} \operatorname{dim} J_{0}(N) .
$$

It is remarkable that the results (65) and (66) can be established unconditionally with almost as good quality. The techniques that achieve this are quite different from and more sophisticated than those used to establish the density results for the family III though both make use of the methods for averaging over such a family, developed in [I-S]. In [DU], Duke examines the averages of $L\left(\frac{1}{2}, f\right)$ and $L^{2}\left(\frac{1}{2}, f\right)$ over the set $H(N)$. This allows him to show that at least $N /(\log N)^{2}$ of 
the $L\left(\frac{1}{2}, f\right)$ 's are non-zero. Introducing "mollifiers" and other tools into analysis of weighted averaging of $L\left(\frac{1}{2}, f\right)$ and $L^{2}\left(\frac{1}{2}, f\right)$, Iwaniec and Sarnak [I-S] show that given $\epsilon_{0}>0$ there is an effective $N_{0}=N_{0}\left(\epsilon_{0}\right)$ such that for $N>N_{0}$

$$
\frac{\#\left\{f \in H^{+}(N) \mid L\left(\frac{1}{2}, f\right) \geq(\log N)^{-2}\right\}}{\#\left\{f \in H^{+}(N)\right\}} \geq \frac{1}{2}-\epsilon_{0} .
$$

This unconditional result is rather close to the conditional (65) and the $50 \%$ in (68) is of fundamental significance. For it is shown in [I-S] that if (68) holds with any $c>\frac{1}{2}$ replacing $\frac{1}{2}$, then there are no Siegel zeroes! (A Siegel zero is a zero of a Dirichlet $L$-function $L\left(s, \chi_{q}\right), \chi_{q}^{2}=1$ which is very close, in terms of $q$ the conductor of $\chi$, to 1.) The precise result that $c>\frac{1}{2}$ would yield is that there is an effective $c^{\prime}>0$ such that $L\left(1, \chi_{q}\right) \geq c^{\prime}(\log q)^{-2}$.

Using variations of the techniques above among many other ideas, Kowalski and Michel [K-M1] and independently VanderKam [V] have shown that for $N$ large

$$
\operatorname{rank} J_{0}(N) \geq B \operatorname{dim} J_{0}(N)
$$

([K-M1] show that $B=19 / 54$ works while $[\mathrm{V}]$ establishes the result with $B=$ 1/100). In another work, Kowalski and Michel [K-M2] establish the upper bound in (62) unconditionally with 99/100 replaced by 10 .

We conclude this section with a comment about more general families $\mathcal{F}$ and the distribution of low-lying zeroes. While symmetry alone appears to dictate the laws of this distribution for the families discussed in Section 4, some caution must be exercised in general. For example, consider the family $\mathcal{F}$ of isogeny classes of all elliptic curves $E / \mathbb{Q}$ ordered by their conductors. Function field analogues (as in example 2 of Section 4 ) suggest that the symmetry $G(\mathcal{F})$ of this family is $O$. In particular (and this can be proven for the function field with the usual caveat of letting $q \rightarrow \infty$ first), zero percent of the $E$ 's in $\mathcal{F}$ of conductor at most $N$ have rank at least two, as $N \rightarrow \infty$. However, numerical experimentation with moderate size $N$ for this family and some other families of elliptic curves [K-Z], [SI2], [B-M] indicate that this percentage is positive and even that it is not very small. It is premature to say whether this is an artifact of a too restricted range of computation and is due to effects like there being points of small height on these curves (like the Gouvea-Mazur result [GO-MA]), or whether the distribution of low-lying zeroes for such a family does not follow the $O$-predictions. Understanding the source of this "excess rank" for moderate $N$ will no doubt reveal some very interesting new features.

\section{Conclusion}

Phenomenologically, it is found that the distribution of the high zeroes of any $L$-function follow the universal GUE Laws, while the distribution of the low-lying zeroes of certain families follow the laws dictated by symmetries associated with the family. The function field analogues of these phenomena can be established, and the source of the symmetry is the monodromy of the family and its scaling limits. Analytic results concerning the distribution of high zeroes for an individual $L$-function and low zeroes of a family of $L$-functions, to the extent to which these can be established, confirm these findings above.

Whether in the case of $L$-functions (over $\mathbb{Q}$ ) there is indeed some kind of underlying monodromy group which glues the family and is the source of the symmetry is a fascinating question. Our belief is that there is. One can imagine that to each 
$L(s, f), f \in \mathcal{F}$, there is a natural interpretation of the zeroes of $L(s, f)$ as the eigenvalues of an operator $U(f)$ on some space $H$. As $f$ varies over $\mathcal{F}$ these $U(f)$ 's become equi-distributed in the space of such operators with a given symmetry type. For the families discussed in Section 4 these symmetries are identified. In particular, the Riemann Zeta function sits in Family I of Section 4 which has a symplectic symmetry. We infer that in the proposed spectral interpretation of the zeroes of the Riemann Zeta function, the operator should preserve a symplectic form! ${ }^{4}$ We believe that the further understanding of the source of such symmetries holds the key to finding a natural spectral interpretation of the zeroes.

\section{REFERENCES}

[A-Z] A. Altland, M. Zirnbauer, "Non-Standard Symmetry Classes in Mesoscopic NormalSuper-Conducting Hybrid Structures," Cond-Mat/9602137.

[AR] E. Artin, "Quadratische Körper in Geibiet der Höheren Kongruzzen I and II," Math. Zeit., 19, 153-296, (1924).

[BO] E. Bombieri, "Hilbert's 8-th Problem an Analogue," Proc. Sym. Pure Math. AMS XXVIII, 269-275, (1976). MR 55:2913

[BR] A. Brumer, "The Rank of $J_{0}(N)$," Asterisque, 228, 41-68, (1995). MR 96f:11083

[B-HB] A. Brumer, R. Heath-Brown, "The Average Rank of Elliptic Curves II," (preprint), (1992).

[B-K] E. Bogomolny, J. Keating, "Random matrix theory and the Riemann zeros. I. Threeand four-point correlations," J. Non-linearity, 8, 1115, (1995). MR 97d:11132a

[B-M] A. Brumer, O. McGuinness, "The Behavior of the Mordell-Weil Group of Elliptic Curves," Bull. AMS, 23, 375-382, (1990). MR 91b:11076

[B-S] B. Birch, P. Swinnerton-Dyer, "Notes on Elliptic Curves (I) and (II)," J. Reine Angew Math., 212, 7-25, (1963), 28, 79-108, (1965). MR 26:3669; MR 31:3419

[CO] A. Connes, "Formule de Trace en G'eometrie Non-Commutative et Hypothesis de Riemann," CR. Acad. Sci., Paris, 323, I, 1231-1236, (1996). MR 97k:11124

[DA] H. Davenport, "Multiplicative Number Theory," Springer Verlag, G.T.M., (1974). MR 82m:10001

[DE] P. Deligne, "La Conjecture de Weil I and II," Publ. I.H.E.S., 43, 273-307, (1974), 52, 313-428, (1981). MR 49:5013; MR 83c: 14017

[DEN] C. Denninger, "Evidence for a Cohomological Approach to Analytic Number Theory," First European Math Congress, Vol. 1, (1992), 491-510, Birkhauser, (1994).

[D-F-I] W. Duke, J. Friedlander, H. Iwaniec, "Representations by the Determinant and Mean Values of $L$-Functions," in Sieve Methods, Exponential Sums and their Applications in Number Theory, Cambridge University Press, 109-115, (1997). CMP 98:15

[DU] W. Duke, "The Critical Order of Vanishing of Automorphic L-Functions with Large Level," Invent. Math., 119, 165-174, (1995). MR 95k:11075

[DY] F. Dyson, "Statistical Theory of Energy Levels III," J. Math. Phys., 3, 166-175, (1962). MR 26:1113

[FE] W. Feller, "Introduction to Probability Theory and its Applications," John Wiley, Vol. II, (1966). MR 35:1048

[GA] M. Gaudin, "Sur la loi Limite de L'espacement de Valuers Propres D'une Matrics Aleatiore," Nucl. Phys., 25, 447-458, (1961).

[GO] D. Goldfeld, "Conjectures on Elliptic Curves over Quadratic Number Fields," in Number Theory Conference, Carbondale \& Spanger, L-N-M, 751, 108-118, (1979). MR 81i: 12014

[GO-MA] F. Gouvea, B. Mazur "The square free sieve and the rank of elliptic curves", JAMS 4, (1991), No. 1, 1-23. MR 92b:11039

[GO-MO] D. Goldston, H. Montgomery, "Pair Correlation of Zeros and Primes in Short Intervals," Progress in Math., Vol. 70, Birkhauser, 183-203, (1987). MR 90h:11084

\footnotetext{
${ }^{4}$ Apparently the cohomological formalism of Denniger [DEN] accounts for the "operator" corresponding $\zeta(s)$ preserving a skew-symmetric form.
} 
[G-J] S. Gelbart, H. Jacquet, "A Relation Between Automorphic Representations of GL(2) and GL(3)," Ann. Sci. Ecole Norm. Sup 4, 11, 471-542, (1978). MR 81e:10025

[G-M] M. Gaudin, M. Mehta, "On the Density of Eigenvalues of a Random Matrix," Nucl. Phys., 18, 420-427, (1960). MR 22:3741

[G-Z] B. Gross, D. Zagier, "Heegner Points and Derivatives of L-Series," Invent. Math., 84, 225-320, (1986). MR 87j:11057

[HB] D. R. Heath-Brown, "The Size of Selmer Groups for the Congruent Number Problem II," Invent. Math., 118, 331-370, (1994). MR 95h:11064

[HE] D. Hejhal, "On the Triple Correlation of the Zeroes of the Zeta Function," IMRN, 293-302, (1994). MR 96d:11093

[HEL] S. Helgason, "Differential Geometry, Lie Groups and Symmetric Spaces," Academic Press, New York, (1978). MR 80k:53081

[IW] H. Iwaniec, "Topics in Analytic Number Theory," Rutgers University Course, (1988).

[I-L-S] H. Iwaniec, W. Luo, P. Sarnak, "Low Lying Zeroes of Families of $L$-Functions," (preprint), (1998).

[I-S] H. Iwaniec, P. Sarnak, "The Non-Vanishing of Central Values of Automorphic LFunctions and Siegel's Zero," (preprint), (1997).

[JA] H. Jacquet, "Principal L-Functions of the Linear Group," Proc. Sym. Pure Math., Vol. 33, A.M.S., Part 2, 63-86, (1979). MR 81f:22029

[KA1] N. Katz, "An Overview of Deligne's Work on Hilbert's Twenty-First Problem," Proc. Pure Math. AMS XXVIII, 537-557, (1976). MR 55:5627

[KA2] N. Katz, "Big Twists Have Big Monodromy" (in preparation), (1998).

[KA3] N. Katz, "Affine Cohomological Transforms, Perversity, and Monodromy", JAMS 6, (1993), No. 1, 149-222. MR 94b:14013

[K-L] V. Kolyvagin and D. Lugachev, "Finiteness of the Shafarevich-Tate Group and the Group of Rational Points for some Modular Abelian Varieties," Leningrad Math. J., 1, No. 5, 1229-1253, (1990). MR 91c:11032

[K-M1] E. Kowalski, P. Michel, "Sur de Rang de $J_{0}(N)$," (preprint), (1997).

[K-M2] E. Kowalski, P. Michel, "Sur les Zeros de Fonctions l Automorphes de Grand Niveau," (preprint), (1997).

[K-S1] N. Katz, P. Sarnak, "Random Matrices, Frobenius Eigenvalue and Monodromy," AMS Colloq. series (to appear), (1999).

[K-S2] N. Katz, P. Sarnak, "Zeroes of Zeta Functions, their Spaces and their Spectral Nature," (1997 preprint version of the present paper).

[K-Z] G. Kramarz, D. Zagier, "Numerical Investigations Related to $L$-Series of Certain Elliptic Curves," J. Indian Math. Soc., 52, 51-69, (1987). MR 90d:11072

[LA] R. Langlands, "Problems in the Theory of Automorphic Forms," Springer, L.N.M., 170, 18-86, (1970). MR 46:1758

[MA] B. Mazur, "Modular Curves and the Eisenstein Ideal," Publ. Math. I.H.E.S., 47, 33186, (1977). MR 80c: 14015

[MEH] M. Mehta, "Random Matrices," Second Edition, Academic Press, Boston (1991). MR 92f: 82002

[MER] L. Merel, "Bornes pour la Torsion de Courbes Elliptiques sur les Corps de Nombres," Invent. Math., 124, 437-449, (1996). MR 96i:11057

[MO1] H. Montgomery, "Topics in Multiplicative Number Theory," L.N.M., 227, Springer, (1971). MR 49:2616

[MO2] H. Montgomery, "The Pair Correlation of Zeroes of the Zeta Function," Proc. Sym. Pure Math., 24, AMS, 181-193, (1973). MR 49:2590

[MU] M. Murty, "The Analytic Rank of $J_{0}(N) / Q$," Number Theory, (Halifax, NS), CMS Conf. Proc., AMS, 15, 263-277, (1995). MR 96i:11054

[OD] A. Odlyzko, "The $10^{20}$-th Zero of the Riemann Zeta Function and 70 Million of its Neighbors," (preprint), A.T.T., (1989).

[O-S] A. Ozluk, C. Snyder, "Small Zeroes of Quadratic L-Functions," Bull. Aust. Math. Soc., 47, 307-319, (1993). MR 94c:11080

[P-P] A. Perelli, J. Pomykala, "Averages Over Twisted Elliptic L-Functions," Acta Arith., 80, 149-163, (1997). MR 98c: 11044

[P-S] R. Phillips, P. Sarnak, "On Cusp Forms for Cofinite Subgroups of $P S L(2, \mathbb{R})$," Invent. Math., 80, 339-364, (1985). MR 86m:11037 
[RI] B. Riemann, "Über die Anzahl der Primzahlen uter Einer Gegebenen Gröbe," Montasb. der Berliner Akad., (1858160), 671-680.

[RUB] M. Rubinstein, "Evidence for a Spectral Interpretation of Zeros of $L$-Functions," Thesis, Princeton University, (1998).

[RUM] R. Rumely, "Numerical Computations Concerning ERH," Math. Comp., 61, 415-440, (1993). MR 94b:11085

[R-S] Z. Rudnick, P. Sarnak, "Zeros of Principal L-Functions and Random Matrix Theory," Duke Math. Jnl., 81, 2, 269-322, (1996). MR 97f:11074

[SA] P. Sarnak, "L-Functions," ICM Talk, Berlin, 1998.

$[\mathrm{SCH}] \quad$ F.K. Schmidt, "Analytische Zahlen Theorie in Körpen der Charakteristik p," Math. Zeit., 33, 1-32, (1931).

[SH1] G. Shimura, "On the Holomorphy of Certain Dirichlet Series," Proc. London Math. Soc. (3), 31, 79-98, (1975). MR 52:3064

[SH2] G. Shimura, "On Modular Forms of Half-Integral Weight," Ann. Math., 97, 440-481, (1973). MR 48:10989

[SI1] J. Silverman, "The Arithmetic of Elliptic Curves," G.T.M., Springer, (1986). MR 87g:11070

[SI2] J. Silverman, "The Average Rank of a Family of Elliptic Curves," (preprint), (1997).

[ST] S.A. Stepanov, "The Number of Points of Hyperelliptic Curve Over a Prime Field," Izv. Akad. Nauk. Sssr. Mat., 33, 1171-1181, (1969). MR 40:5620

[TA $\quad J$. Tate, "On the Conjecture of Birch Swinnerton-Dyer and a Geometric Analogue," Seminar Bourbaki, Fev, (1966, Exp. 306). CMP 98:09

[TI] E. Titchmarsh, "The Theory of the Riemann Zeta Function," Oxford University Press, Second Edition, (1986). MR 88c:11049

[V] J. Vanderkam, "The Rank of Quotients of $J_{0}(N)$," (preprint), (1997).

[WE1] A. Weil, "Über die Bestimmung Dirichletscher Reihen Durch Funktion Algleichungen," Math. Ann., 168, 149-156, (1967). MR 34:7473

[WE2] A. Weil, "Basic Number Theory," Third Edition, Springer, Berlin, (1974). MR 55:302

[WE3] A. Weil, "Sur les Functions Algebriques à corps de Constantes Fini," C.R.Acad. Sci., Paris, 210, 592-594, (1940). MR 2:123d

[WE4] A. Weil, "On the Riemann Hypothesis in Function Fields," Proc. Nat. Acad. Sci., U.S.A., 27, 345-349, (1941). MR 2:345b

[WI] E. Wigner, "Random Matrices in Physics," Siam Review, 9, 1-23, (1967).

[WO] S. Wong, "Rank Zero Twists of Elliptic Curves," (preprint), (1996).

Department of Mathematics, Princeton University, Fine Hall, Washington Road, Princeton, New Jersey 08544

E-mail address: nmk@math.princeton.edu

E-mail address: sarnak@math.princeton.edu 